\title{
On vortex shedding from an airfoil in low-Reynolds-number flows
}

\author{
SERHIY YARUSEVYCH ${ }^{1} \dagger$, PIERRE E. SULLIVA N \\ AND JOHN G. KA W A LL $L^{3}$ \\ ${ }^{1}$ Department of Mechanical \& Mechatronics Engineering, University of Waterloo, Waterloo, \\ N2L 3G1, Canada \\ ${ }^{2}$ Department of Mechanical and Industrial Engineering, University of Toronto, Toronto, \\ M5S 3G8, Canada \\ ${ }^{3}$ Department of Mechanical and Industrial Engineering, Ryerson University, Toronto, M5B 2K3, Canada
}

(Received 22 May 2008 and in revised form 23 February 2009)

Development of coherent structures in the separated shear layer and wake of an airfoil in low-Reynolds-number flows was studied experimentally for a range of airfoil chord Reynolds numbers, $55 \times 10^{3} \leqslant R e_{c} \leqslant 210 \times 10^{3}$, and three angles of attack, $\alpha=0^{\circ}, 5^{\circ}$ and $10^{\circ}$. To illustrate the effect of separated shear layer development on the characteristics of coherent structures, experiments were conducted for two flow regimes common to airfoil operation at low Reynolds numbers: (i) boundary layer separation without reattachment and (ii) separation bubble formation. The results demonstrate that rollup vortices form in the separated shear layer due to the amplification of natural disturbances, and these structures play a key role in flow transition to turbulence. The final stage of transition in the separated shear layer, associated with the growth of a sub-harmonic component of fundamental disturbances, is linked to the merging of the roll-up vortices. Turbulent wake vortex shedding is shown to occur for both flow regimes investigated. Each of the two flow regimes produces distinctly different characteristics of the roll-up and wake vortices. The study focuses on frequency scaling of the investigated coherent structures and the effect of flow regime on the frequency scaling. Analysis of the results and available data from previous experiments shows that the fundamental frequency of the shear layer vortices exhibits a power law dependency on the Reynolds number for both flow regimes. In contrast, the wake vortex shedding frequency is shown to vary linearly with the Reynolds number. An alternative frequency scaling is proposed, which results in a good collapse of experimental data across the investigated range of Reynolds numbers.

\section{Introduction}

In a growing number of new miniaturized mechanical systems, such as small-scale wind turbines and unmanned aerial vehicles, lifting surfaces operate at relatively low airfoil chord Reynolds numbers, i.e. $R e_{c}<500000$. Airfoil operation at low Reynolds numbers differs significantly from that typical for high-Reynolds-number flows (e.g. Tani 1964; Carmichael 1981; Mueller \& DeLaurier 2003). In particular, a laminar boundary layer on the upper surface of the airfoil often separates and forms a separated shear layer. The presence of laminar boundary layer separation has a significant detrimental effect on airfoil performance, affecting airfoil lift and drag. The 

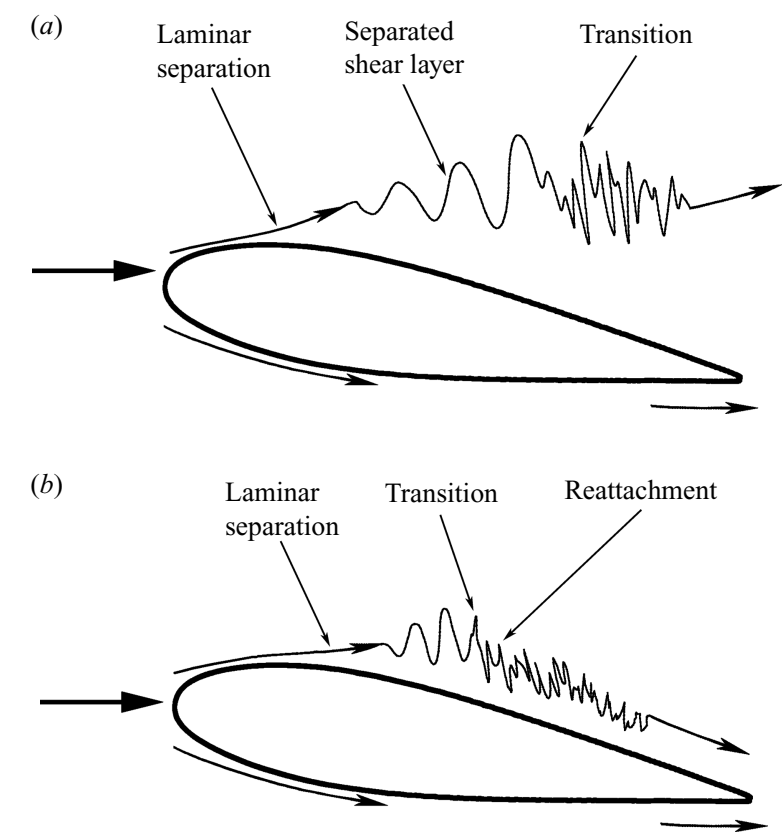

FIGURE 1. Flow over an airfoil at low Reynolds numbers: (a) laminar separation without reattachment; $(b)$ separation bubble formation.

severity of this effect is mainly determined by the behaviour of the separated shear layer. Figure 1 depicts two flow regimes common to airfoils operating at low Reynolds numbers. As the inherently unstable separated shear layer undergoes laminar-toturbulent transition, it can reattach to the airfoil surface. At lower Reynolds numbers, the separated shear layer fails to reattach, and a wide wake is formed (figure $1 a$ ). In contrast, at higher Reynolds numbers, a turbulent separated shear layer may reattach, resulting in a laminar separation bubble (figure $1 b$ ). A change between the two flow regimes depicted in figure 1 is an unsteady phenomenon that occurs over a finite range of Reynolds numbers for a given angle of attack (e.g. Carmichael 1981).

Since the pioneering research into airfoil operation at low Reynolds numbers, summarized and extended by Tani (1964) and Gaster (1967), a number of related studies have been performed over the past several decades. For conciseness, the following discussion of previous studies is focused on those most pertinent to the development of coherent structures in the separated shear layer and airfoil wake.

As illustrated in figure 1, the laminar-to-turbulent transition in the separated shear layer plays a key role in the overall flow field development over an airfoil operating at low Reynolds numbers. Although most of the previous studies dealing with separated shear layer development were performed for a separation bubble forming on a flat plate in an adverse pressure gradient rather than on an airfoil surface, they provide valuable insight into the transition process. It has been shown that, during the initial stage of transition, small-amplitude disturbances centred at some fundamental frequency experience nearly exponential growth in the separated shear layer (e.g. Dovgal, Kozlov \& Michalke 1994; Watmuff 1999; Boiko et al. 2002). Experimental and numerical studies by Haggmark, Bakchinov \& Alfredsson (2000), Lang, Rist \& Wagner (2004), Marxen, Rist \& Wagner (2004) and Marxen \& Rist (2005) suggest 
that two-dimensional growth of the disturbances dominates the initial stage of the transition. The final stage of transition, which results in rapid flow breakdown to turbulence, is associated with nonlinear interactions between the disturbances. Some studies show that coherent structures form during this stage of transition (e.g. Wilson \& Pauley 1998; Watmuff 1999; Lang et al. 2004; Marxen \& Rist 2005, McAuliffe \& Yaras 2007). Specifically, Watmuff suggests that these structures are associated with the Kelvin-Helmholtz instability and persist into the attached turbulent boundary layer. In contrast, experimental results by Lang et al. and direct numerical simulations by Marxen \& Rist and McAuliffe \& Yaras indicate that vortices forming in the separated shear layer breakdown in the reattachment region.

Separated shear layer transition on an airfoil has not been as thoroughly examined as that on a flat plate in an adverse pressure gradient. Nevertheless, the available results obtained for various airfoil profiles indicate that the initial stage of transition is similar to that observed on a flat plate (e.g. Brendel \& Mueller 1988; Brendel \& Mueller 1990; Boiko et al. 2002; Yarusevych, Sullivan \& Kawall 2006). Following the initial linear growth of disturbances, in some studies, nonlinear effects were shown to be associated with the growth of the sub-harmonic of the fundamental frequency wave (e.g. Brendel \& Mueller 1990; Dovgal et al. 1994; Boiko et al. 2002), but the underlying mechanism has not been investigated. The numerical results of Lin \& Pauley (1996), supported by the experimental results and stability analysis of Yarusevych et al. (2006), suggest that coherent structures can form in the separated shear layer and are attributed to the Kelvin-Helmholtz instability (cf. Watmuff 1999). Several other experimental studies on airfoils (e.g. Brendel \& Mueller 1988; Hsiao, Liu \& Tang 1989) found evidence of coherent structures forming during the transition process; however, the behaviour and characteristics of these structures were not investigated in detail. Recently, Burgmann, Brucker \& Schroder (2006), Burgmann, Dannemann \& Schroder (2008) and Burgmann \& Schroder (2008) performed detailed experimental investigations of flow development within a separation bubble on an airfoil. The results identified the roll-up vortices forming in the separated shear layer, which is supported by the results of Zhang, Hain \& Kahler (2008) on the same airfoil profile. Agreeing with the numerical findings of McAuliffe \& Yaras (2007), Burgmann et al. (2008) and Burgmann \& Schroder (2008) suggest that these spanwise structures break down and change orientation in the vicinity of the reattachment point. However, the observed process was found to be different from that reported by Watmuff (1999) and Lang et al. (2004) for a flat-plate bubble. Burgmann et al. (2008) concluded that varying the adverse pressure gradient on an airfoil surface, e.g. by changing the angle of attack, has a different effect on salient bubble characteristics compared to the effect produced by varying the pressure gradient on a flat plate. Despite these research efforts towards describing the development of coherent structures in the separated shear layer on an airfoil, insight into the role of such structures in the transition process, as well as the effect of other flow parameters, such as the Reynolds number, on their characteristics, remains limited. Also, the effect of the flow regime on the development of these structures is uncertain, as most of the previous studies were concerned with the case of a separation bubble. For the case of flow separation without reattachment, some insight into separated shear layer development can be gained from studies on circular cylinders (e.g. Unal \& Rockwell 1988; Prasad \& Williamson 1997). However, in view of the significant differences in geometry, these results cannot be applied directly to airfoils operating at nominally pre-stall angles of attack. 
The development of coherent structures in the airfoil wake at low Reynolds numbers is of interest since it affects airfoil performance and governs flow around downstream objects. For instance, these structures can result in undesirable structural vibrations and noise generation. The wake of an airfoil at post-stall angles of attack can be expected to behave similar to that of a bluff body (e.g. Huang et al. 2001). The structure and characteristics of a two-dimensional bluff-body wake have been the subject of active research over the past decades (e.g. Roshko 1993; Williamson 1996). A comprehensive description of the vortex formation mechanism in the wake of a circular cylinder is presented by Gerrard (1966). The wake vortex shedding frequency is usually scaled with global parameters to form a Strouhal number $S t$, with typical values of 0.21 and 0.14 reported for the case of a circular cylinder and a flat plate, respectively (e.g. Roshko 1954b). For a NACA 0012 airfoil in the range of chord Reynolds numbers from about $25 \times 10^{3}$ to $120 \times 10^{3}$ at angles of attack above about $15^{\circ}$, Huang \& Lin (1995) measured a constant Strouhal number $S t_{d}$ based on the length of the airfoil projection on a cross-stream plane. In agreement with the results of Roshko (1954b), an increase of angle of attack above about $15^{\circ}$ resulted in a decrease of Strouhal number, with an $S t_{d}$ value of 0.12 obtained at $90^{\circ}$.

At lower angles of attack, even when separation occurs without reattachment, airfoil wake characteristics have been shown to be quite different. Huang \& Lin (1995) and Huang \& Lee (2000) detected vortex shedding in the airfoil wake at low Reynolds numbers and identified several vortex shedding modes, with a wide distribution of Strouhal numbers observed over the investigated Reynolds number range. These modes were found to be closely related to separated shear layer behaviour, and wake vortex shedding was observed only when laminar separation occurred without subsequent reattachment or in the presence of turbulent boundary layer separation. In contrast, Yarusevych et al. (2006) also detected organized structures in the airfoil wake for the case when a separation bubble formed on the airfoil surface. Huang et al. (2001) proposed empirical correlations for the dimensionless vortex shedding frequency in the wake of an impulsively started wing for $R e_{c}<2500$. Several other studies confirm the existence of the wake vortex shedding phenomenon for airfoils in low-Reynolds-number flows but did not investigate it in detail (e.g. Williams-Stuber \& Gharib 1990; Gerontakos \& Lee 2005). It should be noted that there are limited results available for vortex shedding in airfoil wakes compared to those concerned with bluff-body wakes. Moreover, evolution of coherent structures in the airfoil wake and the effect of separated shear layer development on their characteristics have not been investigated in detail.

The present work is motivated by the need for additional insight into coherent structures forming in low-Reynolds-number flows over airfoils. The main goal is to investigate the formation, evolution, and characteristics of the coherent structures and their role in the overall flow field development for both flow regimes typical of airfoil operation at low Reynolds numbers. In the following sections, the experimental approach is discussed first. Then, a brief overview of the flow development is provided for the cases investigated. The main results detailing formation, characteristics and frequency scaling of coherent structures are discussed in two sections: (i) coherent structures in the separated shear layer and (ii) coherent structures in the airfoil wake.

\section{Experimental setup}

All experiments were performed in a low-turbulence recirculating wind tunnel. The 5 -m-long test section of this tunnel has a spanwise extent of $0.91 \mathrm{~m}$ and a height of 
$1.22 \mathrm{~m}$. The free-stream turbulence intensity level in the test section is less than $0.1 \%$, and there is no significant frequency-centred activity associated with the oncoming flow. During the experiments, the free-stream velocity $U_{0}$ was monitored by a Pitot tube, with an uncertainty estimated to be less than $2.5 \%$.

An aluminium NACA 0025 airfoil with a chord length $c$ of $0.3 \mathrm{~m}$ was examined. This NACA profile was selected because it allowed investigating both strongly separated flows and separation bubbles at nominally pre-stall angles of attack and the Reynolds numbers of interest. The airfoil was mounted horizontally in the test section $0.4 \mathrm{~m}$ downstream of the wind-tunnel contraction, spanning the entire width of the test section. With this arrangement, it was verified experimentally that end effects did not influence flow development over at least $50 \%$ of the airfoil span within the domain of interest. The angle of attack was set by a digital protractor, with an uncertainty of $0.1^{\circ}$

To enable surface pressure measurements, the airfoil was equipped with 65 pressure taps, $0.8 \mathrm{~mm}$ in diameter, which were positioned at the midspan symmetrically on the upper and lower surfaces. Surface pressure distributions were measured with a pressure transducer connected to the taps through a 64-channel Scanivalve module. The uncertainty associated with the surface pressure measurements was less than $2 \%$.

Flow velocity data were obtained with Dantec constant temperature anemometers. A normal hot-wire probe, a cross-wire probe and a rake of three cross-wire probes were used in separate measurements. The probes were attached to a holder mounted on a remotely controlled traversing mechanism. The mechanism allowed probe motion in the vertical $y$ and streamwise $x$ directions with a resolution of $0.01 \mathrm{~mm}$ and $0.25 \mathrm{~mm}$, respectively. For boundary layer measurements, the probe holder could be manually adjusted to change the angle between the probe and the airfoil surface. To minimize possible probe interference, this angle was kept below $7^{\circ} \pm 0.1^{\circ}$, as recommended by Brendel \& Mueller (1988). All hot-wire measurements were carried out in the vertical midspan plane of the tunnel. Based on the results of Kawall, Shokr \& Keffer (1983), the maximum hot-wire measurement error was evaluated to be less than $5 \%$. The origin of the streamwise coordinate $x$ was located at the leading edge of the airfoil. For boundary layer measurements, the vertical coordinate $y$ was referenced to the airfoil surface; whereas, for wake measurements, the vertical coordinate was referenced to the trailing edge. It should be noted that a conventional single hotwire probe is incapable of determining flow direction and, therefore, cannot resolve the velocity direction of the reverse flow that occurs near the airfoil surface in the separated flow region. However, hot-wire measurements in the separated shear layer, which is of particular interest in the present study, can be analysed without any restrictions.

Spectral analysis of velocity signals was performed to uncover organized flow structures and determine their characteristics. Autospectra of the velocity signals were determined by means of the fast Fourier transform algorithm applied to the experimental data. To allow adequate comparison of the velocity spectra, the velocity data at each streamwise location were collected at a $y / c$ position that corresponds to the maximum r.m.s velocity. Such a position approximately corresponds to the location of half the boundary layer edge velocity $U_{e}$ in the separated shear layer and half the maximum velocity deficit in the wake. Each spectrum was normalized by the variance of the sampled signal, so that the area under the spectral curve was unity. The uncertainty of the spectral analysis was approximately $4.5 \%$, with a frequency resolution bandwidth of $1.2 \mathrm{~Hz}$. 
A smoke-wire technique was employed for flow visualization. The following three smoke-wire configurations were utilized: (i) a single wire installed $15 \mathrm{~cm}$ upstream of the leading edge, (ii) a single wire installed $3 \mathrm{~mm}$ downstream of the trailing edge and (iii) the combination of these two wires. Each wire was coated with smoke-generator fluid, and the fluid was evaporated by electrically heating the wire. A $0.076 \mathrm{~mm}$ diameter stainless steel wire was chosen in order to provide adequate smoke density, while not introducing measurable disturbances into the flow field. The flow was illuminated with a remotely triggered speedlight, i.e. a high-speed flash, positioned in the far wake and the images were obtained with a digital camera. The camera acquired four consecutive images per second with an image resolution of 6 mega pixels.

\section{Overview of flow development}

Experiments were conducted for a range of Reynolds numbers, $55 \times 10^{3} \leqslant$ $R e_{c} \leqslant 210 \times 10^{3}$, and three angles of attack, $\alpha=0^{\circ}, 5^{\circ}$ and $10^{\circ}$. Flow visualization was performed to gain a basic understanding of the boundary layer and wake development. Representative results obtained at $\alpha=5^{\circ}$ and three Reynolds numbers $R e_{c}=55 \times 10^{3}, R e_{c}=100 \times 10^{3}$ and $R e_{c}=150 \times 10^{3}$ are shown in figures $2-4$. Each figure presents two images obtained with different single smoke-wire arrangements to provide a more comprehensive overview of flow development over the airfoil. The results correspond to two distinct flow regimes: (i) boundary layer separation without reattachment for $R e_{c}=55 \times 10^{3}$ and $R e_{c}=100 \times 10^{3}$, and (ii) flow in the presence of the separation bubble on the upper surface of the airfoil for $R_{c}=150 \times 10^{3}$. For $R e_{c}=55 \times 10^{3}$ and $R e_{c}=100 \times 10^{3}$, the boundary layer on the upper surface of the airfoil separates at approximately $x / c=0.25$, and a wide wake is formed. In contrast, for $R e_{c}=150 \times 10^{3}$, the separated shear layer reattaches and remains attached at the trailing edge, as smoke from the downstream smoke wire does not propagate upstream (figure 4). As confirmed by surface pressure measurements, a separation bubble forms on the upper surface of the airfoil for this combination of the Reynolds number and the angle of attack. Evidently, the two identified boundary layer flow regimes are associated with distinctly different wake characteristics. For the two lower Reynolds numbers, large-scale vortices form in the airfoil wake (figures 2 and 3). On the other hand, for $R e_{c}=150 \times 10^{3}$ (figure 4), the scale of wake structures is noticeably smaller and the structures appear to be less organized.

The Reynolds number effect on the boundary layer behaviour on the upper surface of the airfoil is depicted in figure 5, which shows surface pressure distributions for a range of Reynolds numbers at $\alpha=5^{\circ}$. In this figure, $C_{p}=\left(p-p_{0}\right) /\left(0.5 \rho U_{0}^{2}\right)$, where $p$ is the static pressure on the airfoil surface, $p_{0}$ is the static pressure in the free stream, $\rho$ is the density of air and $U_{0}$ is the free-stream velocity in the test section. In these surface pressure distributions, the presence of the separation region can be identified from the region of nearly constant static pressure (Tani 1964). For $R e_{c}=200 \times 10^{3}$, a separation bubble is formed on the upper surface between $x / c=0.45$ and 0.58 . As the Reynolds number decreases to $150 \times 10^{3}$, the separation bubble broadens slightly, located between $x / c=0.4$ and 0.6 , resulting in a diminishment of the suction peak that occurs close to the leading edge $(x / c \approx 0.2)$. Further decrease of the Reynolds number brings about significant changes in the boundary layer development. For $R e_{c}=143 \times 10^{3}$, the boundary layer on the upper surface separates at approximately $x / c=0.35$ and fails to reattach, despite evidence of transition, marked by a mild pressure recovery past $x / c=0.55$. This abrupt increase in the size of the separation 
(a)

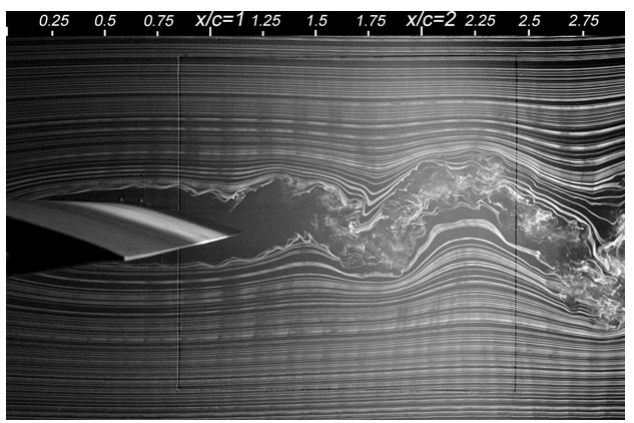

(b)

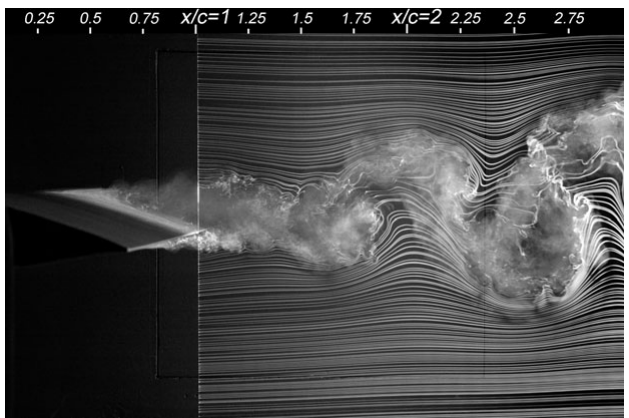

FIGURE 2. Flow visualization for $R e_{c}=55 \times 10^{3}$ at $\alpha=5^{\circ}:(a)$ upstream smoke wire;

(b) downstream smoke wire.

(a)

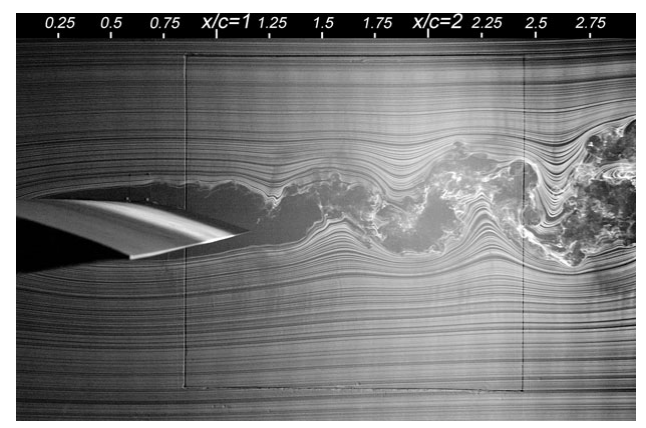

(b)

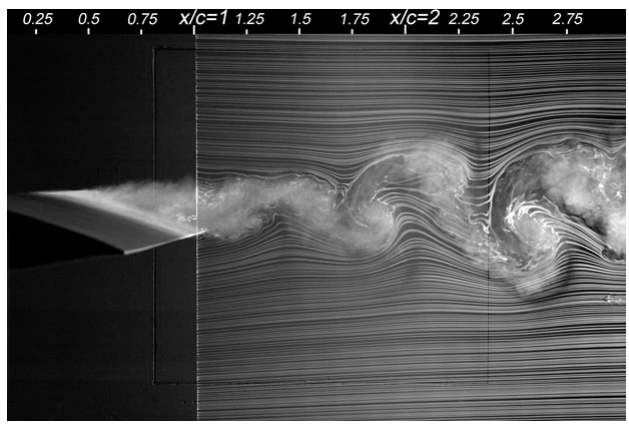

FIGURE 3. Flow visualization for $R e_{c}=100 \times 10^{3}$ at $\alpha=5^{\circ}:(a)$ upstream smoke wire; (b) downstream smoke wire.

(a)

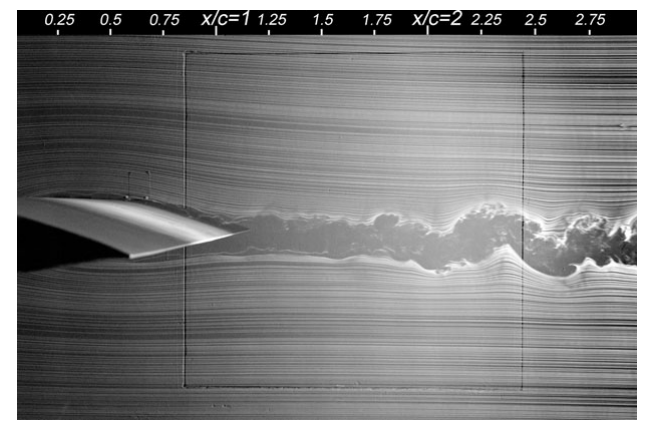

(b)

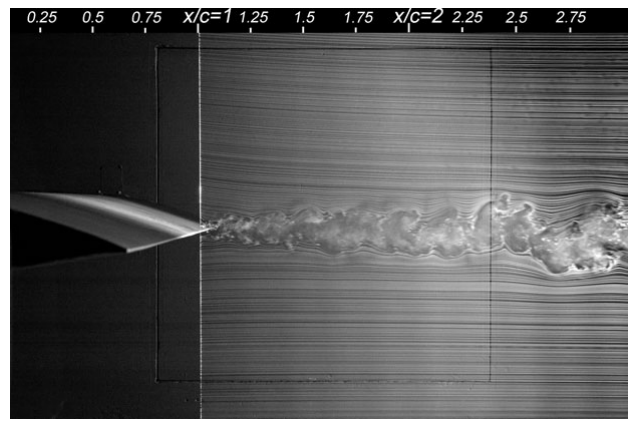

FIGURE 4. Flow visualization for $R e_{c}=150 \times 10^{3}$ at $\alpha=5^{\circ}:(a)$ upstream smoke wire; (b) downstream smoke wire.

region is often referred to as bubble bursting and results in a significant reduction of the suction peak on the upper surface, stalling the airfoil. Eventually, as the Reynolds number decreases to $135 \times 10^{3}$, the boundary layer on the upper surface separates at $x / c=0.3$, forming a wake. Further decrease of the Reynolds number does not appreciably affect the pressure distribution. 
Boundary layer separation $\alpha \quad$ without subsequent reattachment

$\begin{aligned} 0^{\circ} & 55 \times 10^{3} \leqslant R e_{c} \leqslant 110 \times 10^{3} \\ 5^{\circ} & 55 \times 10^{3} \leqslant R e_{c} \leqslant 125 \times 10^{3} \\ 10^{\circ} & 55 \times 10^{3} \leqslant R e_{c} \leqslant 135 \times 10^{3}\end{aligned}$

Separation bubble

$135 \times 10^{3} \leqslant R e_{c} \leqslant 210 \times 10^{3}$

$150 \times 10^{3} \leqslant R e_{c} \leqslant 210 \times 10^{3}$

$175 \times 10^{3} \leqslant R e_{c} \leqslant 210 \times 10^{3}$

TABLE 1. Boundary layer flow regimes for $55 \times 10^{3} \leqslant R e_{c} \leqslant 210 \times 10^{3}$.

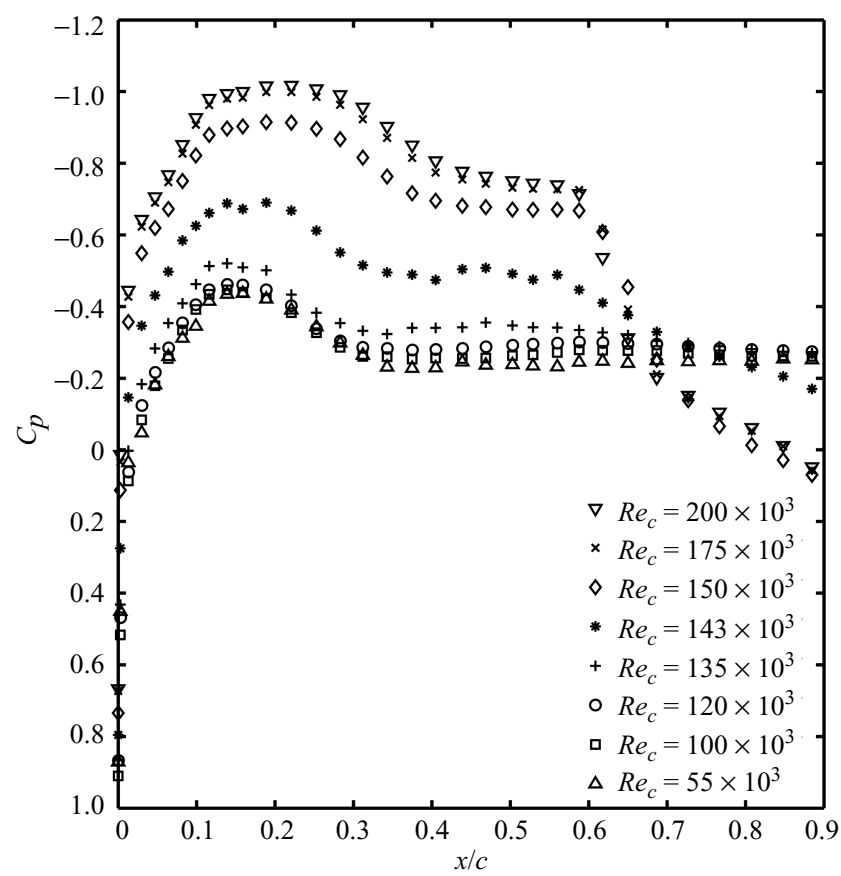

FIGURE 5. Reynolds number effect on surface pressure distributions on the upper surface at $\alpha=5^{\circ}$.

For the angles of attack investigated, surface pressure and boundary layer velocity measurements (Yarusevych 2006) were utilized to characterize boundary layer behaviour for the cases investigated. These results are summarized in table 1, which details the extent of the two flow regimes. As reflected in table 1, there is a short transitional region between the two regimes at each angle of attack, where bubble bursting/reattachment can occur sporadically due to high sensitivity of the flow to changes in experimental parameters. Since the boundaries of this region are difficult to determine precisely, a conservative approach was employed, with the corresponding boundaries in table 1 overestimated by up to $10 \times 10^{3}$.

\section{Coherent structures in separated shear layer}

\subsection{Roll-up vortices and their role in flow transition}

The laminar-to-turbulent transition process in the separated shear layer is depicted in figure 6, which shows spectra of the separated shear layer velocity data on the upper surface of the airfoil for $R e_{c}=55 \times 10^{3}, R e_{c}=100 \times 10^{3}$ and $R e_{c}=150 \times 10^{3}$ 
(a)

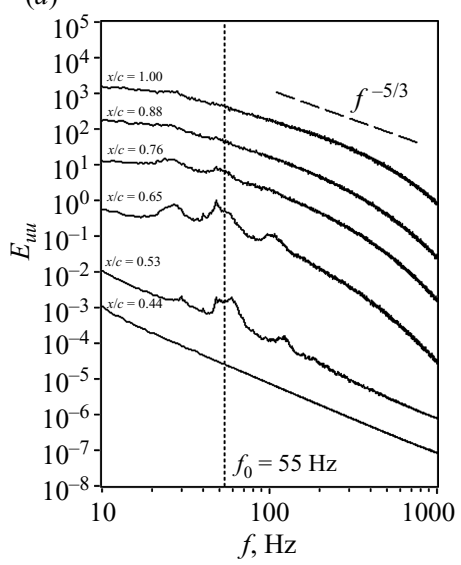

(b)

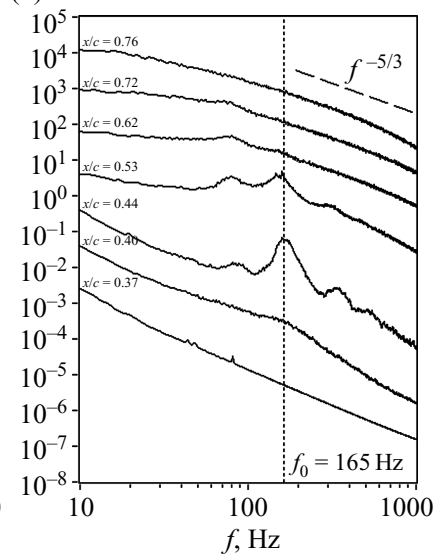

(c)

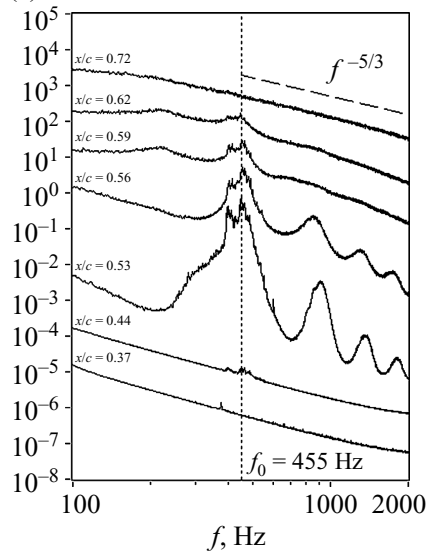

FIGURE 6. Spectra of the streamwise fluctuating velocity component at $\alpha=5^{\circ}$ : (a) $R e_{c}=$ $55 \times 10^{3}$, (b) $R e_{c}=100 \times 10^{3}$ and (c) $R e_{c}=150 \times 10^{3}$. The amplitude of each successive spectrum is stepped by one order of magnitude.

at $\alpha=5^{\circ}$. For clarity, the amplitude of each spectrum is stepped by an order of magnitude with respect to the spectrum at the previous upstream location. Although the presented results pertain to the two different flow regimes, with flow separation without reattachment occurring for $R e_{c}=55 \times 10^{3}$ and $R e_{c}=100 \times 10^{3}$ (table 1), a similar transition mechanism is observed. In particular, disturbances within a band of frequencies, centred at a fundamental frequency $f_{0}$ are amplified in the separated shear layer, with $f_{0}$ increasing as the Reynolds number increases. The initial growth of the disturbances is followed by the generation and growth of harmonics and a subharmonic of the fundamental frequency, which is indicative of nonlinear interactions between the disturbances (Dovgal et al. 1994). This is followed by a rapid laminar-toturbulent transition, with a 'classical' turbulence spectrum observed in the aft portion of the separated flow region. A similar transition mechanism was observed at all the angles of attack investigated.

Coherent structures developing in the separated shear layer are shown in figures 7 and 8, which feature close-up images of the separated flow region at $\alpha=5^{\circ}$ for $R e_{c}=55 \times 10^{3}$ and $R e_{c}=100 \times 10^{3}$, respectively. For $R e_{c}=55 \times 10^{3}$, the results reveal three well-defined vortices in the separated shear layer on the upper surface (figure $7 a$ ), with the first appearing at approximately $x / c=0.5$, and two vortices on the lower surface close to the trailing edge. Four similar vortices can also be identified on the upper surface of the airfoil in figure $7(b)$. It is evident from figure $7(b)$ that the identified vortices form close to the interface between the recirculating flow region and the separated shear layer. As the Reynolds number increases to $R e_{c}=100 \times 10^{3}$ (figure 8), the vortices seem to form earlier upstream and their length scale decreases substantially compared to that for $R e_{c}=55 \times 10^{3}$. It is noted that the formation of the vortices in the separated shear layer at approximately $x / c=0.6$ for $R e_{c}=55 \times 10^{3}$ (figure 7) correlates with the occurrence of the spectral peaks in figure $6(a)$, at $x / c=0.53$ and 0.65 , centred at the fundamental frequency of $55 \mathrm{~Hz}$. As the Reynolds number increases to $100 \times 10^{3}$, the vortices form at approximately $x / c=0.45$ (figure 8 ), correlating with the presence of a strong spectral peak in figure $6(b)$ at $x / c=0.44$ centred at the fundamental frequency of $165 \mathrm{~Hz}$. Furthermore, the downstream spacing of the vortices is approximately constant in the 
(a) (b)
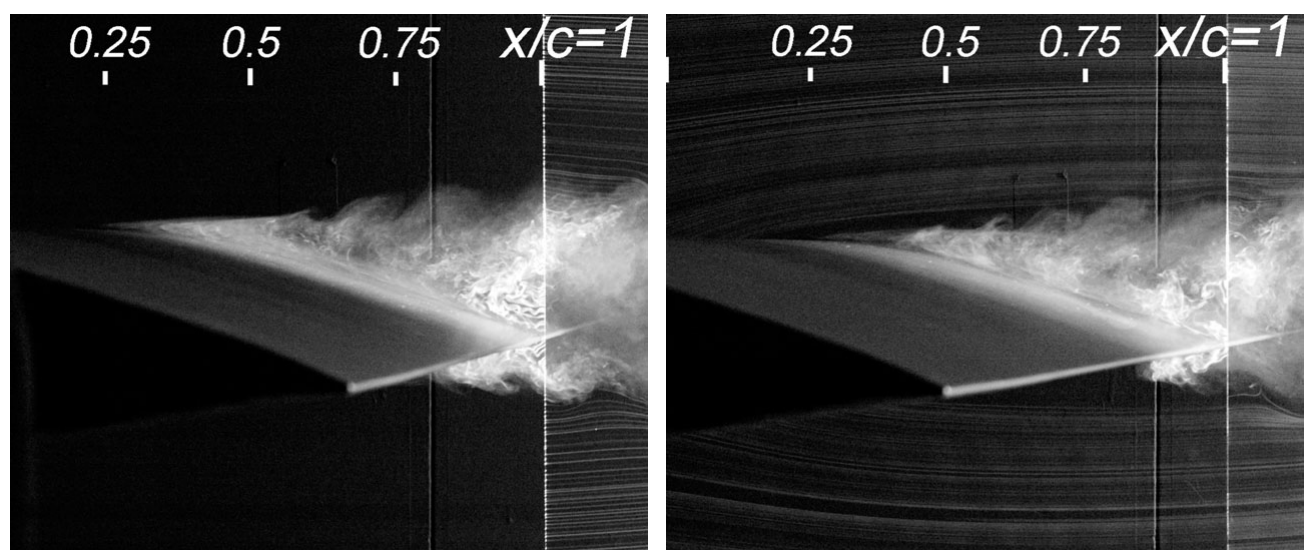

FIGURE 7. Flow visualization of the separated region for $R e_{c}=55 \times 10^{3}$ at $\alpha=5^{\circ}:(a)$ with a single downstream smoke wire; $(b)$ with both upstream and downstream smoke wires.

(a)

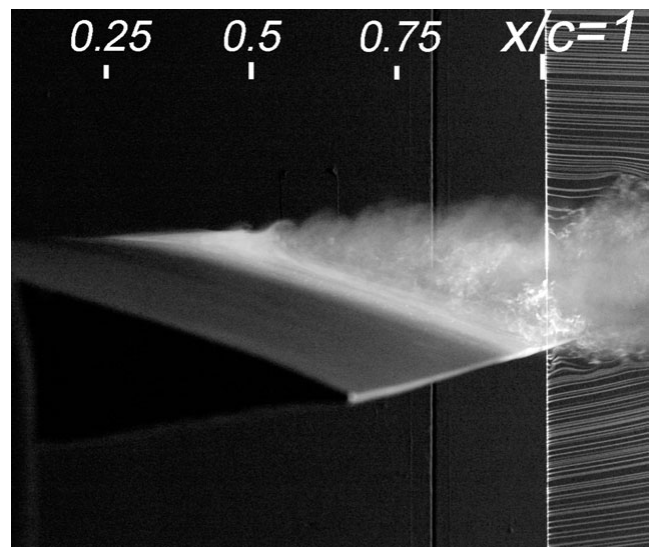

(b)

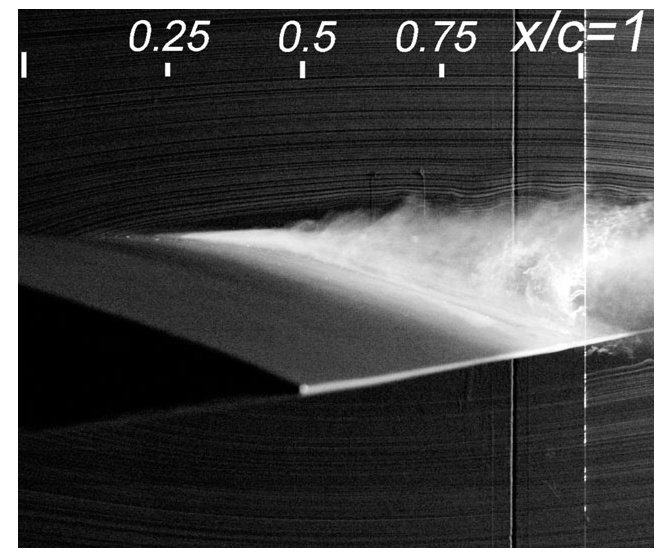

FiguRE 8. Flow visualization of the separated region for $\operatorname{Re}_{c}=100 \times 10^{3}$ at $\alpha=5^{\circ}:(a)$ with a single downstream smoke wire; $(b)$ with both upstream and downstream smoke wires.

corresponding images for $R e_{c}=55 \times 10^{3}$ and $R e_{c}=100 \times 10^{3}$, suggesting that they are shed at a constant frequency for a given Reynolds number. In the absence of other frequency-centred activity in the flow, it can be concluded that the observed shear layer vortices are shed at the fundamental frequency and, hence, are linked to the most amplified flow disturbances. The implication here is that growing periodic disturbances eventually cause the shear layer to roll up, producing roll-up vortices. The observed roll-up process appears to be similar to that common to free shear layers (e.g. Miksad 1972; Huang \& Ho 1990) and was also reported by Watmuff (1999), Lang et al. (2004) and Marxen \& Rist (2005) for the separation bubble induced on a flat plate.

A comparative analysis of the flow visualization images (figures 7 and 8) and velocity spectra (figures $6 a$ and $6 b$ ) indicates that the shear layer transition process is associated with the decay of the roll-up vortices, as no evidence of coherent structures is seen in the spectra past the transition. The observed transition via sub-harmonic growth of the fundamental frequency was also reported in previous 
(a)

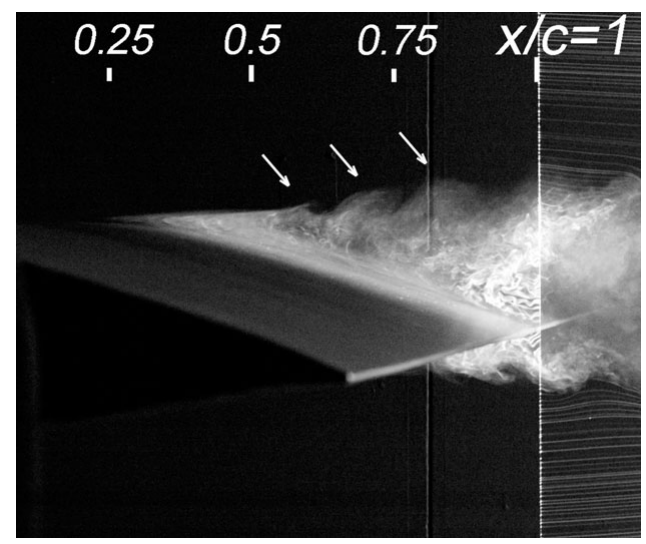

(b)

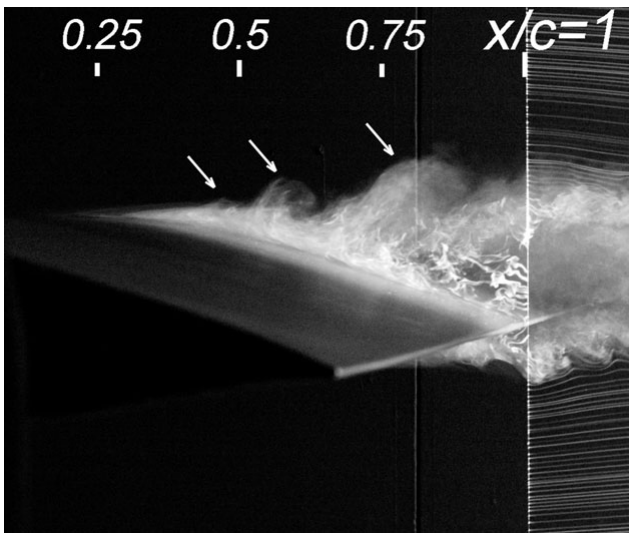

FIGURE 9. Consecutive flow visualization images depicting merging of the shear layer roll-up vortices for $R e_{c}=55 \times 10^{3}$ at $\alpha=5^{\circ}$. Flow visualization with a single downstream smoke wire. Image $(b)$ was taken $0.25 \mathrm{sec}$ after image $(a)$. Roll-up vortices are marked by arrows.

studies on airfoils operating at low Reynolds numbers (e.g. Brendel \& Mueller 1990; Malkiel \& Mayle 1996; Boiko et al. 2002). However, the mechanism responsible for this is not clear. In an experimental study on an airfoil-like model, Malkiel \& Mayle speculated that the sub-harmonic growth of disturbances is attributed to vortex merging. Recently, indirect evidence of vortex merging in the airfoil separated shear layer was also obtained by McAuliffe \& Yaras (2005) via a comparison of raw particle image velocimetry (PIV) images. A detailed analysis of consecutive flow images in the present investigation revealed that vortex merging occurs in the separated shear layer. The phenomenon is depicted in figure 9, which presents two consecutive flow visualization images for $\operatorname{Re}_{c}=55 \times 10^{3}$ at $\alpha=5^{\circ}$. Figure $9(a)$ shows three equally spaced roll-up vortices above the upper surface of the airfoil. In contrast, another three roll-up vortices identified in figure $9(b)$ are not equally spaced. While the downstream distance between the first two vortices in figure $9(b)$ is approximately the same as the spacing in figure $9(a)$, the distance between the second and the third vortices is doubled. This suggests that the third vortex, located at $x / c \approx 0.75$, is formed by two merged roll-up vortices and is shed at half the shedding frequency. The velocity spectrum pertaining to $x / c=0.76$ in figure $6(a)$ displays a peak at $27.5 \mathrm{~Hz}$ which is somewhat more pronounced than a diminishing peak at the fundamental frequency of $55 \mathrm{~Hz}$. This indicates that the nonlinear stage of transition associated with the sub-harmonic growth is attributed to the merging of the roll-up vortices in the separated shear layer, which is followed by a rapid breakdown of the vortices.

Unlike the vortex merging phenomenon common in free shear layers (e.g. Miksad 1972; Huang \& Ho 1990), the merging observed in the present investigation is a weakly periodic process, which is evident from broad peaks centred at half the fundamental frequency in the velocity spectra (figure 6). Abdalla \& Yang (2004) detected vortex merging in a numerical study of a separation bubble forming on a flat plate with a blunt leading edge. In agreement with the present findings, their results suggest that the merged vortices rapidly transform into smaller three-dimensional structures, which resemble classical $\Lambda$ shapes and ribs. A similar three-dimensional breakdown process was also observed by McAuliffe \& Yaras (2007), Burgmann et al. (2008) and Burgmann \& Schroder (2008). It was observed in the present study that 
(a)

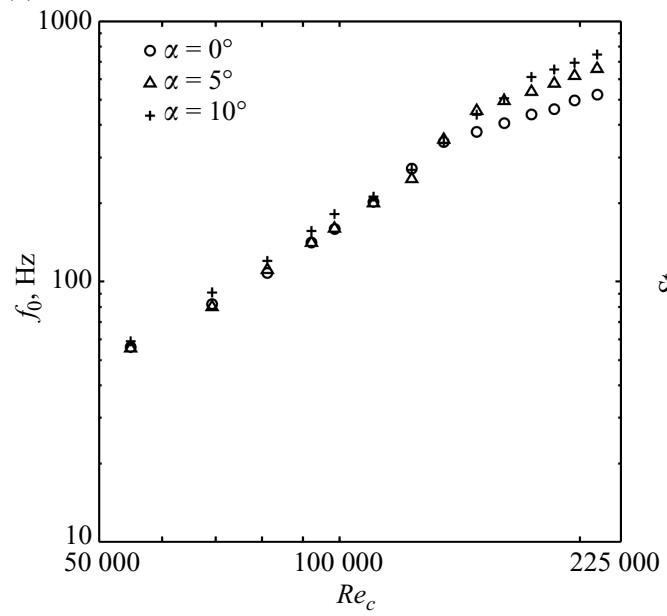

(b)

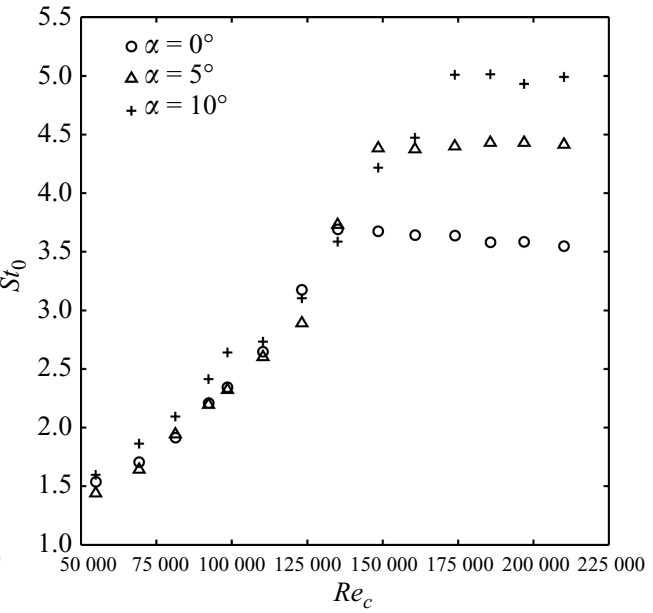

FIGURE 10. Variation of $(a)$ the fundamental frequency of shear-layer disturbances $\left(f_{0}\right)$ and

$(b)$ the corresponding Strouhal number $\left(S t_{0}\right)$ with Reynolds number and angle of attack.

the sub-harmonic growth becomes less pronounced with an increase of the Reynolds number or a decrease of the angle of attack, i.e. as the vertical extent of the separation region at the initial roll-up location decreases. This indicates that the proximity of the wall influences the nonlinear stage of the transition process in the separated shear layer, supporting the observations of Lang et al. (2004) and McAuliffe \& Yaras (2007). Such an influence may be responsible for the fact that vortex merging was not observed in some of the previous investigations.

\subsection{Roll-up frequency scaling}

To investigate the Reynolds number effect on the shedding frequency of the shear layer roll-up in more detail, frequencies of the fundamental disturbances were determined via spectral analysis of velocity measurements. The resulting variation of the fundamental frequency $f_{0}$ and the corresponding Strouhal number, $S t_{0}=f_{0} d / U_{0}$, where $d$ is the length of the airfoil projection on a cross-stream plane, with Reynolds number is presented in figure 10. A comparative analysis of these results and the boundaries of the two flow regimes given in table 1 suggests that the change of the flow regime leads to a significant change in the dependency of the fundamental frequency on the Reynolds number, which is more pronounced in the corresponding plots of the Strouhal number. For boundary layer separation without reattachment, the Strouhal number gradually increases as the Reynolds number increases to approximately $R e_{c}=120 \times 10^{3}$. Also, in this regime, the fundamental frequency does not vary significantly with the angle of attack. In contrast, once the separation bubble is formed on the airfoil surface, the Strouhal number reaches approximately 3.6, 4.4 and 5 at $0^{\circ}, 5^{\circ}$ and $10^{\circ}$, respectively, and remains almost constant with an increase of Reynolds number.

Separate plots for $f_{0}$ at each angle of attack are shown in figure 11. Two distinct sets of data corresponding to the two flow regimes can be identified in each of the plots. Curve fits for these data sets suggest that the fundamental frequency exhibits a powerlaw dependency on the Reynolds number of the form $f_{0} \sim\left(R e_{c}\right)^{n}$. In dimensionless form, this expression becomes $S t_{0} \sim\left(R e_{c}\right)^{n-1}$. Note that data points pertaining to a transitional region between the two regimes (table 1) were not included in the curve 


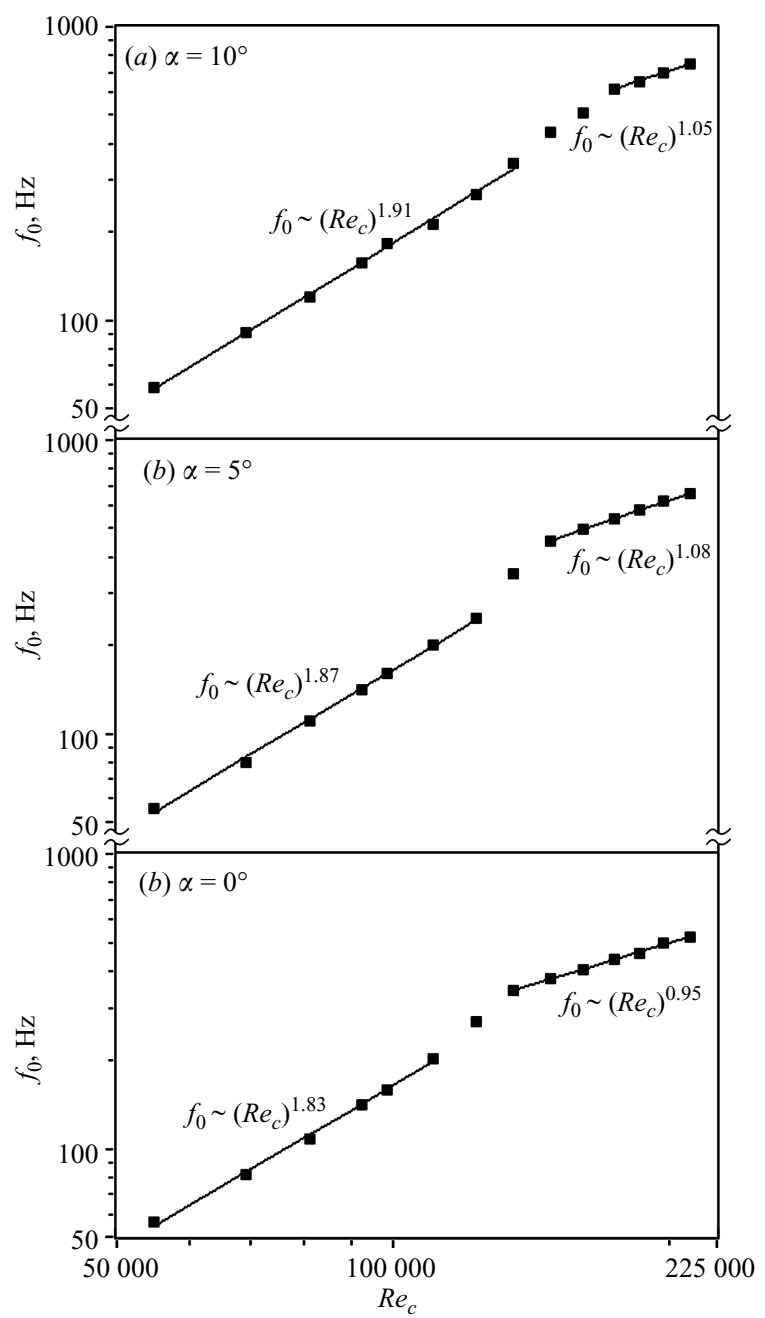

FIGURE 11. Variation of the fundamental frequency of disturbances in the separated shear layer.

fits. A similar power-law dependency between the shear layer roll-up frequency $f_{0}$ and $R e$ can be inferred from previous experimental results for a circular cylinder (e.g. Bloor 1964; Prasad \& Williamson 1997; Thompson \& Hourigan 2005). A power-law fit was applied to available experimental data on airfoil profiles and the results are presented in figure 12. As can be seen from the curve fits in figure 12, the data closely follow the power-law dependency established in the present study. The results in figures 11 and 12 show that the value of the exponent $n$ varies within a relatively wide range of approximately 0.9-1.9. This differs from the results for the circular cylinder, where only a minor variation in $n$ was observed (e.g. Thompson \& Hourigan 2005). The fundamental frequency is expected to scale as $f_{0} \sim U_{e s} / L_{s}$, where $U_{e s}$ is the boundary-layer edge velocity at separation and $L_{s}$ is the characteristic boundary layer length scale at separation, e.g. the boundary layer thickness. In the case of an airfoil, both of these parameters depend on the Reynolds number, angle of attack and airfoil shape, which explains the observed variation in the exponent. 


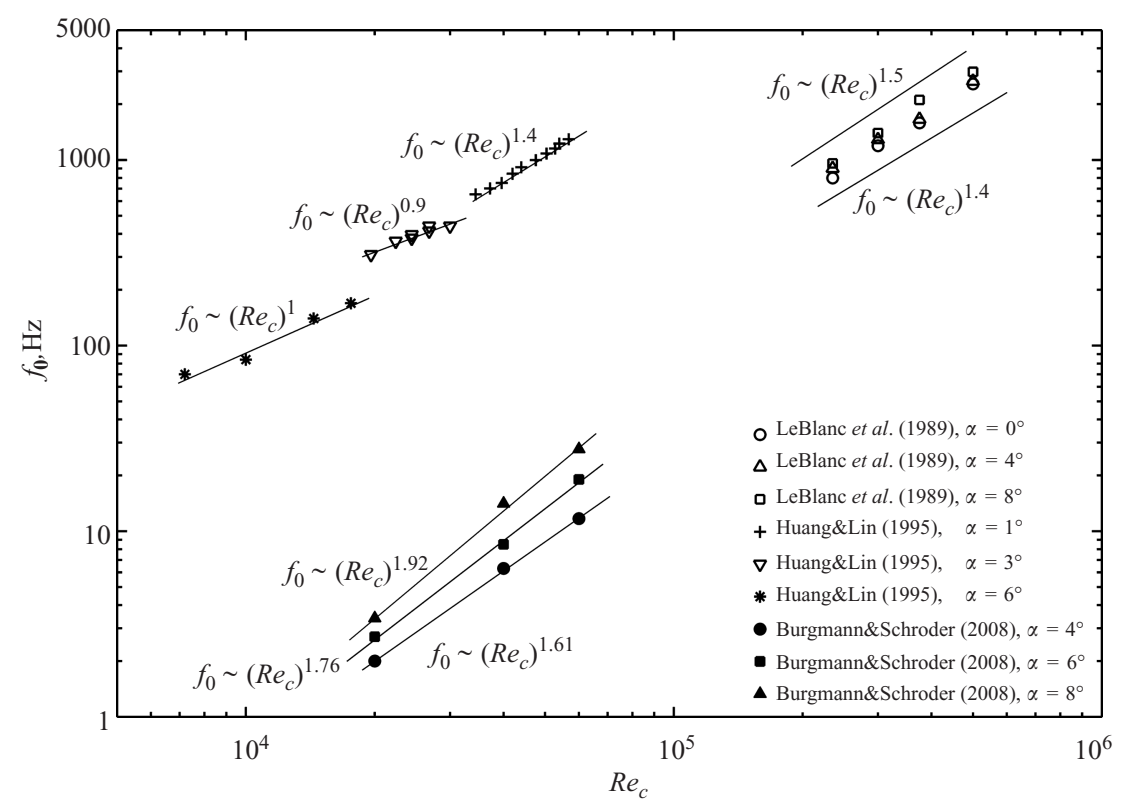

FIgURE 12. Power-law dependency between the fundamental frequency and $R e_{c}$ applied to experimental data from previous studies.

In free shear layers, the Strouhal number is often defined in terms of the fundamental frequency, initial momentum thickness and average velocity. It has been shown by Ho \& Huerre (1984) that the Strouhal number corresponding to the frequency of the most amplified disturbance is about 0.032 . Similar to this approach, the fundamental frequency in separated shear layers is commonly scaled with the momentum thickness and free-stream velocity at separation (e.g. Lin \& Pauley 1996; Watmuff 1999; Yang \& Voke 2001; McAuliffe \& Yaras 2007; Burgmann et al. 2008). However, in the cited publications, the Strouhal number corresponding to the frequency of roll-up vortices was found to vary over a wide range, namely, 0.005-0.011, with uncertainties in establishing the location of separation and estimating momentum thickness contributing to the variation. In light of this, we propose to consider the wavelength of the fundamental disturbance $\lambda_{0}$ as an alternative length scale, which defines the initial streamwise distance between the roll-up vortices. Huang \& Ho (1990) used $\lambda_{0}$ as a scaling parameter in free shear layers and argued that, in forced shear layers, this is a more appropriate scale compared to the initial momentum thickness. Thus, the alternative definition of the Strouhal number becomes $S t_{0}^{*}=f_{0} \lambda_{0} / U_{e s}$. In this relationship, the wavelength $\lambda_{0}$ can be expressed in terms of the propagation speed of the roll-up vortices $U_{\text {drift }}$ and the roll-up frequency $f_{0}$ as $\lambda_{0}=U_{\text {drift }} / f_{0}$, so that $S t_{0}^{*}=U_{\text {drift }} / U_{e s}$. Furthermore, at a given downstream position, the trajectory of the roll-up vortices matches the location of the maximum r.m.s velocity (McAuliffe \& Yaras 2005; Yarusevych 2006; Burgmann et al. 2008), which, as expected, correlates with the inflection point in the mean velocity profile. Thus, the propagation speed of the roll-up vortices can be estimated based on the average velocity in the shear layer $U_{\text {drift }} \approx\left(U_{e}+U_{\text {rev }}\right) / 2$, i.e. the average of the boundary-layer edge velocity $U_{e}$ and the mean velocity in the reverse flow region adjacent to the wall $U_{\text {rev }}$. The velocity of the reverse flow usually does not exceed $10 \%$ of the boundary-layer edge velocity in the laminar and transitional portions of the separated flow region (e.g. Carmichael 


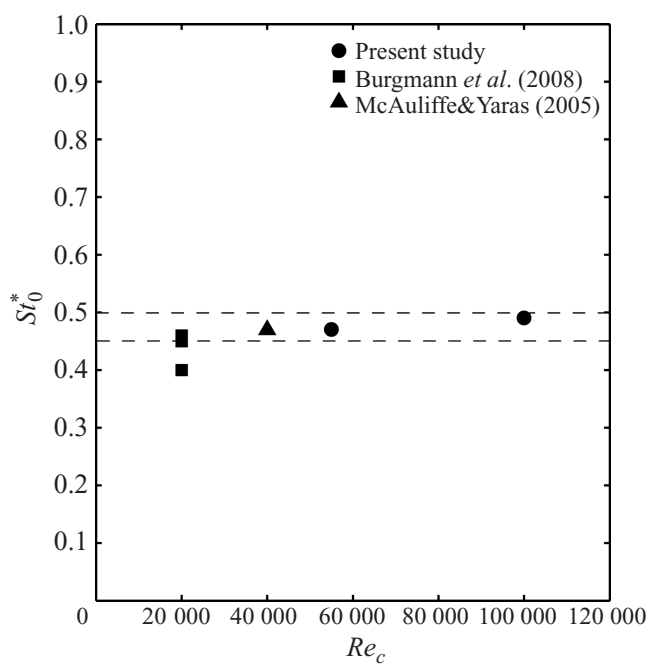

FIGURE 13. Variation of $S t_{0}^{*}$ with chord-based Reynolds number.

1981; Dovgal et al. 1994), and, in the present investigation, was less than $0.05 U_{e}$ for all the cases examined. Thus, for $-0.1 U_{e} \leqslant U_{\text {rev }} \leqslant 0$, the propagation speed of the rollup vortices should fall in the range $0.45 U_{e} \leqslant U_{d r i f t} \leqslant 0.5 U_{e}$. Since the surface pressure remains almost constant in the laminar portion of the separated flow region (e.g. figure 5), the boundary-layer edge velocity in this region, $U_{e} \approx U_{0} \sqrt{1-C_{p}}$, does not significantly deviate from that at separation $U_{e s}$. Therefore, with the proposed scaling, the resulting Strouhal numbers are expected to fall within a relatively narrow range, namely, $0.45 \leqslant S t_{0}^{*} \leqslant 0.5$. Estimating the fundamental frequency from figure 6 and the distance between the roll-up vortices from figures 7 and 8 , we obtain $S t_{0}^{*} \approx 0.47$ for $R e_{c}=55 \times 10^{3}$ at $\alpha=5^{\circ}$ and $S t_{0}^{*} \approx 0.49$ for $R e_{c}=100 \times 10^{3}$ at $\alpha=5^{\circ}$. As can be seen from figure 13, the predicted range of $S t_{0}^{*}$ is in reasonable agreement with these estimates, as well as experimental results obtained for other airfoil models.

\section{Coherent structures in airfoil wake}

\subsection{Wake vortex shedding}

The development of coherent structures in the airfoil wake for the two boundary layer flow regimes investigated is illustrated in figures 14 and 15 , which show consecutive flow visualization images obtained for $R e_{c}=55 \times 10^{3}$ and $R e_{c}=150 \times 10^{3}$ at $\alpha=5^{\circ}$. For $R e_{c}=55 \times 10^{3}$, figure 14 shows large-scale vortices shed alternately into the upper and lower parts of the airfoil wake, forming a pattern similar to that of a Kármán vortex street. As the Reynolds number increases to $150 \times 10^{3}$ and a separation bubble forms on the airfoil surface, the length scale of the wake vortices decreases significantly and the vortex pattern becomes less organized (figure 15). For instance, several vortices located on opposite sides of the wake between $x / c=1.5$ and $x / c=2$ can be identified in figure 15(a), with relatively uniform spacing attributed to an almost constant vortex shedding frequency. However, the vortex pattern in the same downstream region in figure $15(b)$ is difficult to identify, implying that the vortex shedding in this flow regime is a broadband frequency centred activity.

Since spectra of the vertical fluctuating velocity component $E_{v v}$ are more sensitive to frequency-centred activity associated with spanwise structures compared to spectra 
(a) $t=t_{0}$

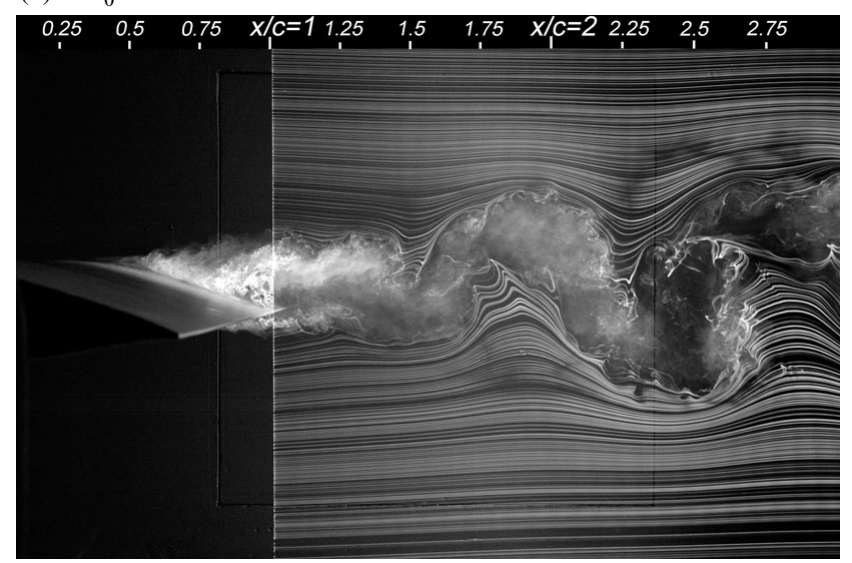

(b) $t=t_{0}+0.25 \mathrm{sec}$

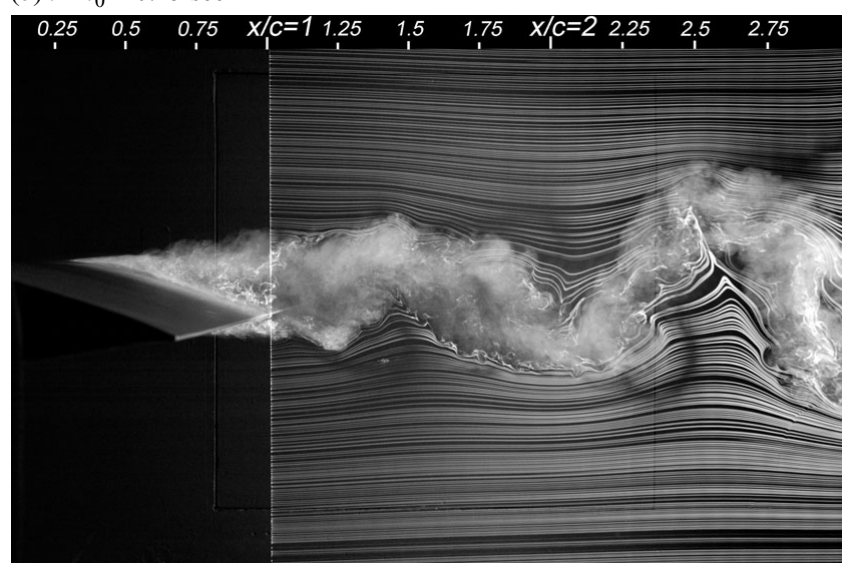

(c) $t=t_{0}+0.5 \mathrm{sec}$

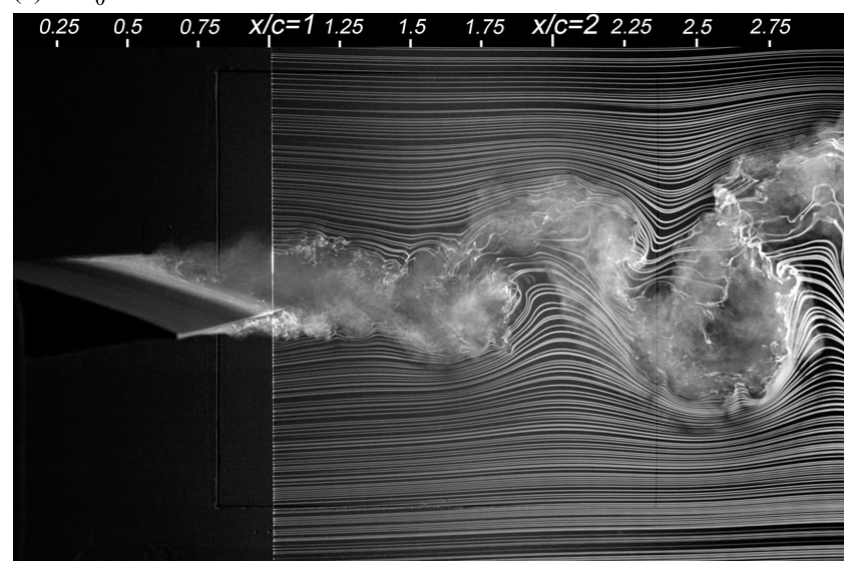

FiguRE 14. Wake vortex shedding visualization for $R e_{c}=55 \times 10^{3}$ at $\alpha=5^{\circ}$.

of the streamwise velocity component $E_{u u}$, a cross-wire probe was employed to investigate the development of coherent structures. Such a probe was not used for boundary layer measurements in close proximity to the separation point due to the relatively large size of the probe compared to the boundary layer thickness. 
(a) $t=t_{0}$

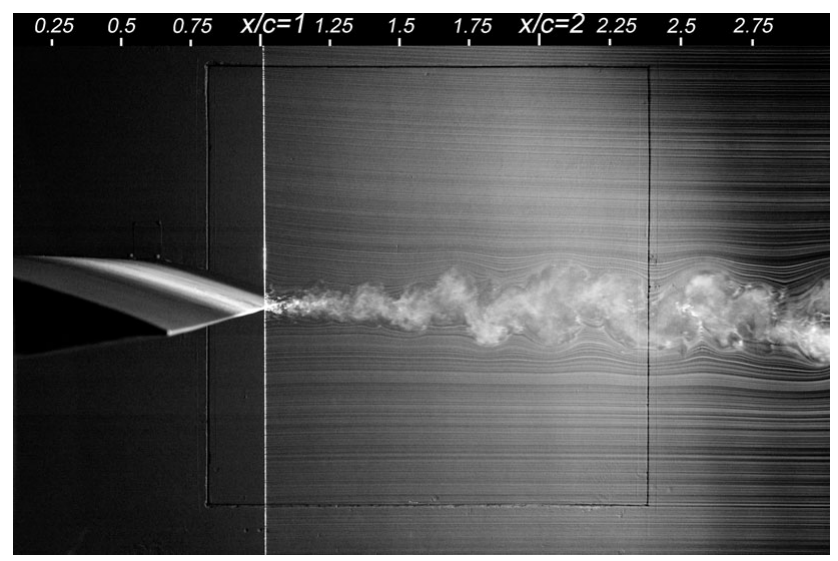

(b) $t=t_{0}+0.25 \mathrm{sec}$

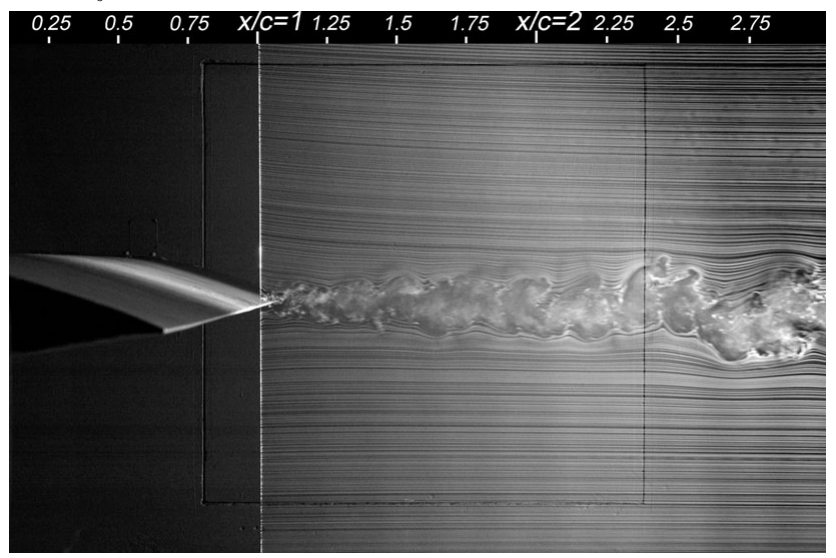

FIGURE 15. Wake vortex shedding visualization for $\operatorname{Re}_{c}=150 \times 10^{3}$ at $\alpha=5^{\circ}$.

Cross-wire measurements were conducted at multiple downstream locations starting in the transition region within the separated shear layer and extending into the airfoil wake. The spectra of the vertical velocity component for $R e_{c}=100 \times 10^{3}$ and $R e_{c}=150 \times 10^{3}$ at $\alpha=5^{\circ}$ are shown in figures $16(a)$ and $16(b)$, respectively. As before, the amplitude of each spectrum has been stepped by an order of magnitude with respect to the spectrum at the previous upstream location. The results in figure 16 correspond to the two flow regimes (table 1) and are representative of all the cases investigated. For both flow regimes, a distinct peak at the fundamental frequency $f_{0}$ occurs in the spectrum due to the shedding of the shear layer roll-up vortices. Subsequent merging of the roll-up vortices produces a second peak in the spectra centred at the sub-harmonic of the fundamental frequency $0.5 f_{0}$. Further downstream, the roll-up vortices quickly break down during the transition process, with no peak associated with the fundamental frequency detectable at and beyond $x / c=0.76$ for both Reynolds numbers. As the turbulent separated shear layer evolves downstream, at $x / c=1$, a peak begins to develop in the spectrum centred at a much lower frequency compared to $f_{0}$. In the near wake, this unambiguous peak is centred at $f_{s}=20 \mathrm{~Hz}$ for $R e_{c}=100 \times 10^{3}$ and $f_{s}=68 \mathrm{~Hz}$ for $R e_{c}=150 \times 10^{3}$. Based on the flow visualization results, it can be concluded that the low-frequency peak in the wake velocity spectra is 
(a)

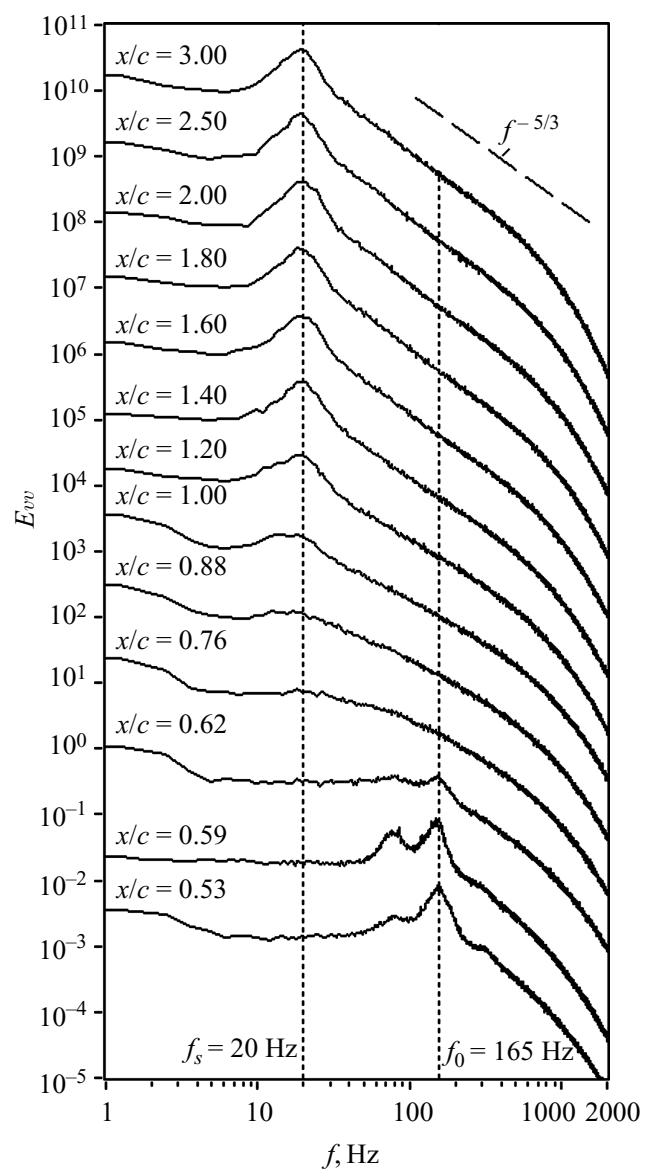

(b)

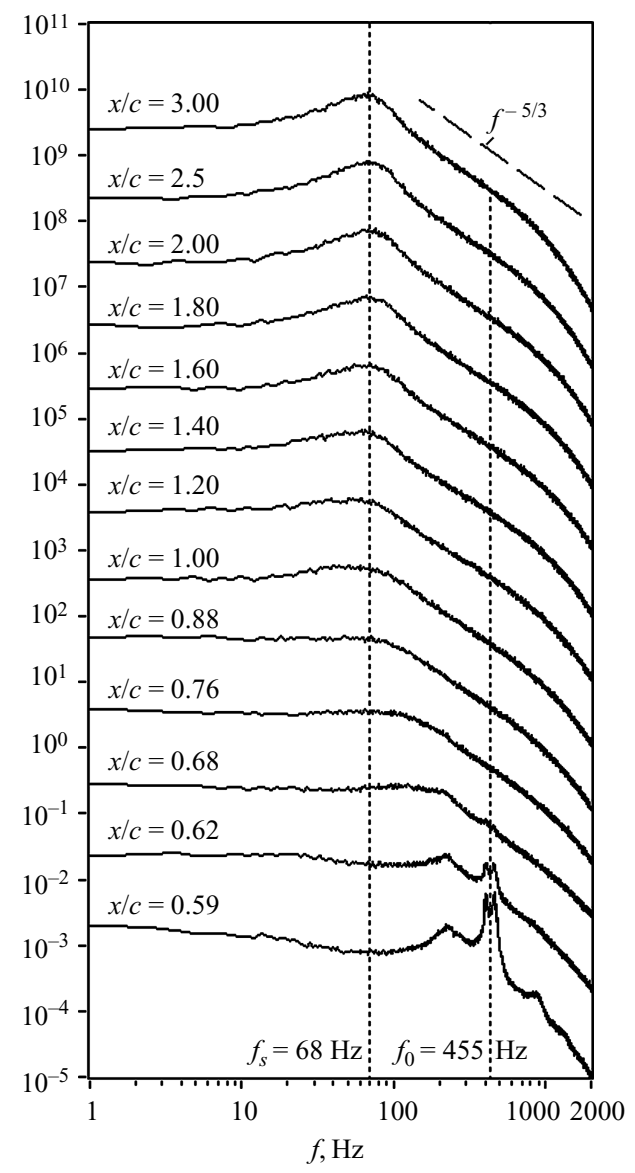

FIGURE 16. Spectra of the vertical fluctuating velocity component at $\alpha=5^{\circ}$ for $(a) R e_{c}=$ $100 \times 10^{3}$ and $(b) R e_{c}=150 \times 10^{3}$. The amplitude of each successive spectrum is stepped by one order of magnitude.

attributed to wake vortex shedding. The downstream growth of the energy content of this frequency-centred activity is checked in the near wake, marking a vortex formation region similar to that observed in the wake of a circular cylinder (e.g. Gerrard 1966; Williamson 1996). This region corresponds to approximately $1 \leqslant x / c \leqslant 1.8$ for $R e_{c}=100 \times 10^{3}$ and to $1 \leqslant x / c \leqslant 2$ for $R e_{c}=150 \times 10^{3}$. Despite the observed similarities in the wake development for the two boundary layer flow regimes, the peaks produced by wake vortex shedding in the spectra for $R e_{c}=150 \times 10^{3}$ (figure $16 b$ ) are broader and less defined compared to those for $R e_{c}=100 \times 10^{3}$ (figure 16a). Agreeing with the flow visualization results, this suggests that the wake vortices produced when a separation bubble forms on the airfoil surface are less coherent than those forming in the airfoil wake when boundary layer separation occurs without reattachment. The presence of coherent structures for both flow regimes investigated was also confirmed via a correlation analysis of velocity signals obtained with a rake of three cross-wire probes, similar to the approach employed by Yarusevych et al. (2006). Significant correlation coefficients substantiated that vortices are shed alternately on opposite sides of the wake in both flow regimes, as observed in flow visualization images. The present results amend the findings of Huang \& Lin (1995), who obtained no evidence 


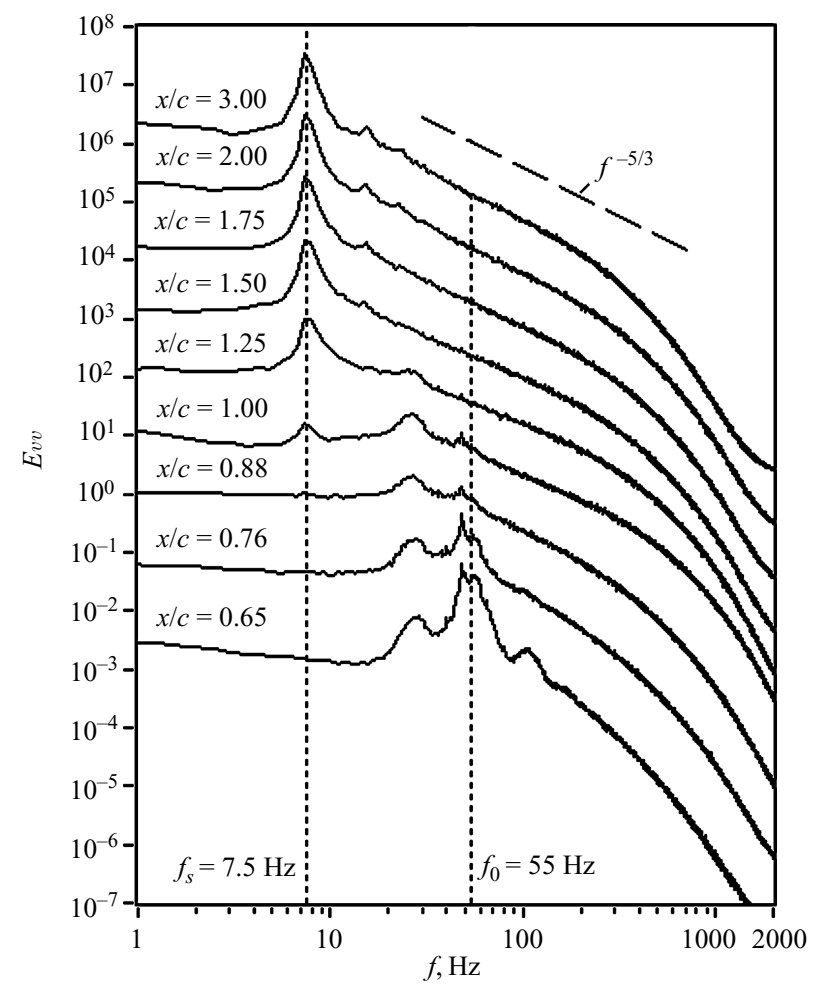

FIGURE 17. Spectra of the vertical fluctuating velocity component for $R e_{c}=55 \times 10^{3}$ at $\alpha=5^{\circ}$. The amplitude of each successive spectrum is stepped by one order of magnitude.

of vortex shedding in the wake of a NACA 0012 airfoil when a separation bubble formed on the airfoil and suggested that this flow regime is not accompanied by vortex shedding. In light of the present findings, however, it is likely that the presence of weaker coherent structures in the airfoil wake in this flow regime was not detected in their investigation because only streamwise velocity signals obtained with a normal hot wire sensor were analysed. Indeed, in the present study, wake vortex shedding in the airfoil wake could not be detected in spectra of the streamwise velocity component and was revealed only in the spectra of the vertical velocity component.

Although the shear layer roll-up vortices break down during the transition process, these vortices may interact with wake vortex shedding if the transition region extends into the near wake. This can take place at lower Reynolds numbers when separation occurs without subsequent flow reattachment, as the transition 'point' tends to shift farther downstream with a decrease of the Reynolds number. Indeed, as evident from the flow visualization results for $R e_{c}=55 \times 10^{3}$ and $R e_{c}=100 \times 10^{3}$ (figures 7 and $8)$, the roll-up vortices in the separated shear layer become more pronounced and propagate further downstream as the Reynolds number decreases. This is illustrated in figure 17, which shows velocity spectra pertaining to $R e_{c}=55 \times 10^{3}$ at $\alpha=5^{\circ}$. Similar to the results for the higher Reynolds numbers, the roll-up vortices produce a peak in the spectra at the fundamental frequency, $f_{0}=55 \mathrm{~Hz}$, and the subsequent merging of these structures is reflected in the growth of the sub-harmonic peak in the spectra obtained downstream of $x / c=0.65$. However, for this Reynolds number, both the sub-harmonic peak and the weak fundamental peak can be identified in the spectrum at $x / c=1$. Some merged vortices persist until about $x / c=1.25$, eventually 


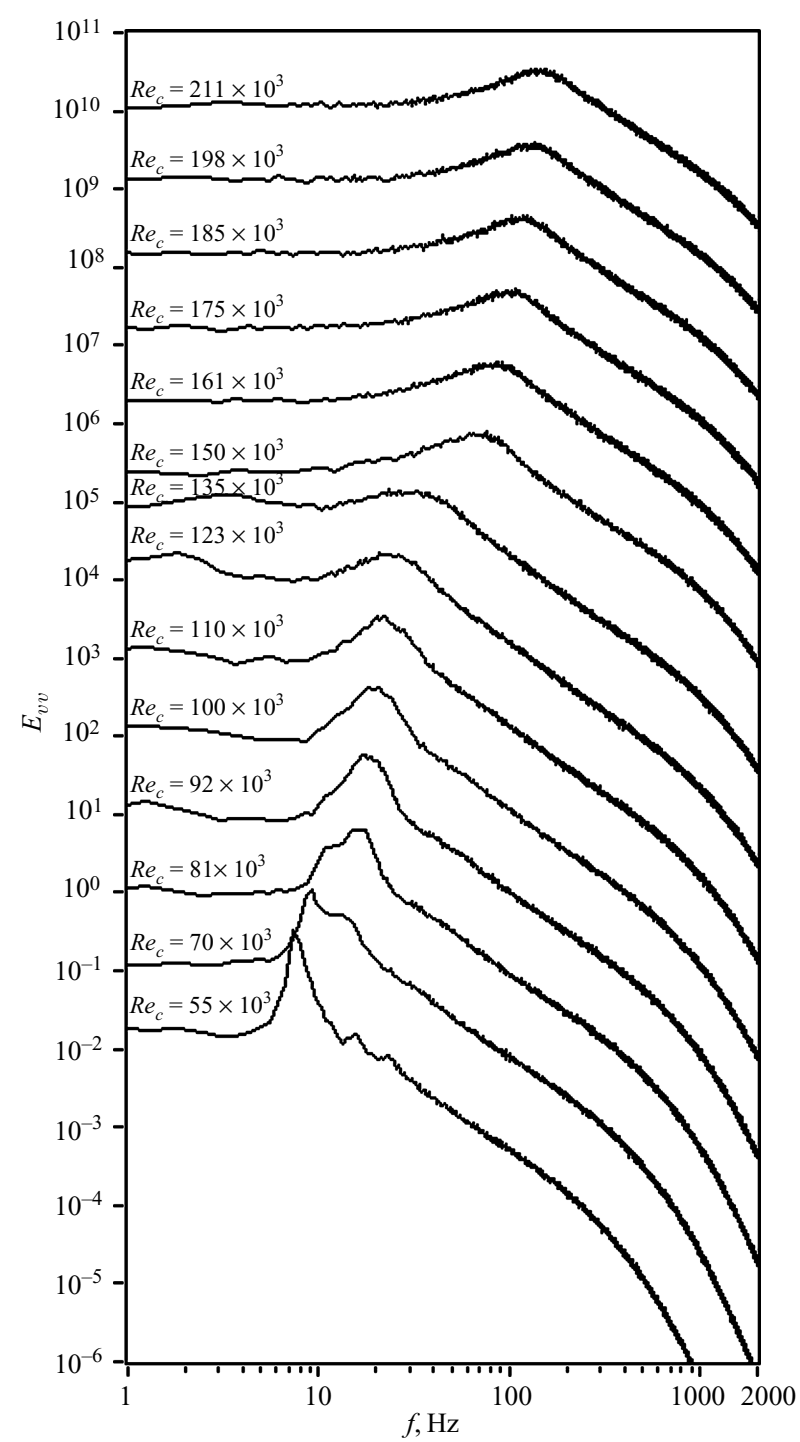

FIGURE 18. Spectra of the vertical fluctuating velocity component at $\alpha=5^{\circ}$ and $x / c=2$. The amplitude of each successive spectrum is stepped by one order of magnitude.

breaking down by $x / c=1.5$. At the same time, wake vortices form in the near-wake region, producing a peak in the corresponding spectra centred at a much lower frequency $f_{s}=7.5 \mathrm{~Hz}$. Thus, it can be expected that shear layer roll-up vortices may interact with wake vortices at lower Reynolds numbers $\left(R e_{c} \leqslant 55 \times 10^{3}\right)$ for which the transition region extends into the near wake. A more detailed examination of this aspect was not carried out since $R e_{c}=55 \times 10^{3}$ represents the lowest attainable Reynolds number for the experimental setup used in the present study.

\subsection{Wake vortex shedding frequency scaling}

The Reynolds number effect on wake vortex shedding characteristics is depicted in figure 18, which shows spectra of the vertical velocity component obtained at $\alpha=5^{\circ}$ and $x / c=2$. A dominant peak attributed to wake vortex shedding occurs in 
(a)

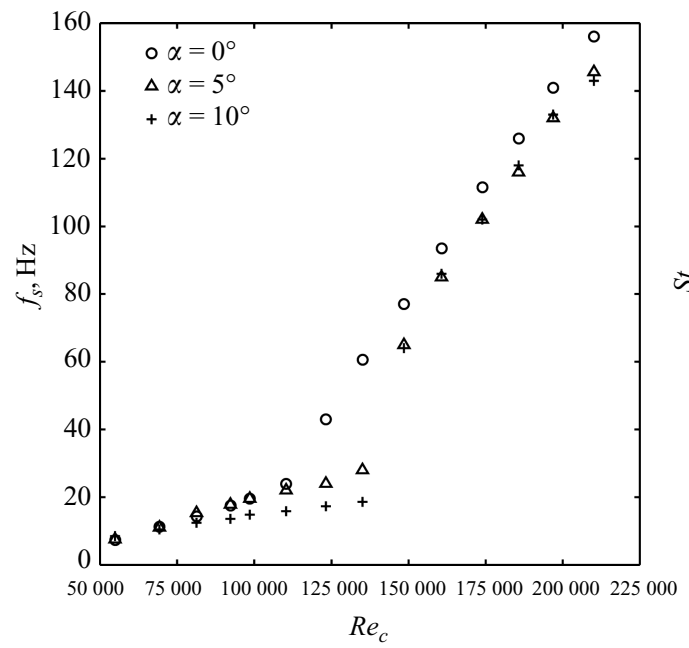

(b)

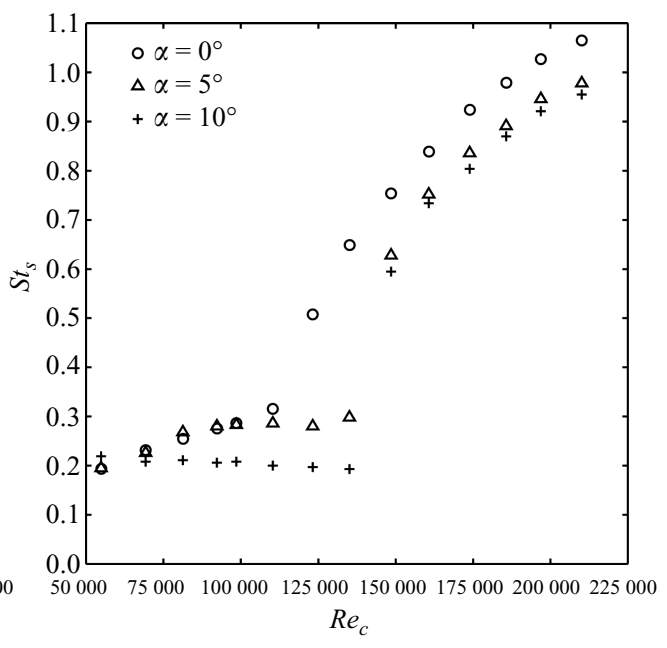

FIGURE 19. Variation of $(a)$ the vortex shedding frequency $\left(f_{s}\right)$ and $(b)$ the corresponding Strouhal number $\left(S t_{s}\right)$ with Reynolds number and angle of attack.

the spectrum for $R e_{c}=55 \times 10^{3}$ at $f_{s}=7.5 \mathrm{~Hz}$. As the Reynolds number increases, the dominant peak shifts to higher frequencies and broadens. Further increase of the Reynolds number eventually leads to boundary layer reattachment between approximately $R e_{c}=123 \times 10^{3}$ and $R e_{c}=135 \times 10^{3}$, producing a dramatic change in vortex shedding characteristics. As a consequence, a sharp increase of the vortex shedding frequency occurs for $R e_{c}=150 \times 10^{3}$. Moreover, the spectral peak broadens, indicating a substantial decrease in coherence of the wake vortices. An increase of the Reynolds number beyond $R e_{c}=150 \times 10^{3}$ is accompanied by a gradual increase of the shedding frequency.

Variation of the wake vortex shedding frequency $f_{s}$ and the corresponding Strouhal number, $S t_{s}=f_{s} d / U_{0}$, with the Reynolds number is shown in figure 19 . The two identified boundary layer regimes are associated with distinctly different trends in the data. In agreement with previous results, the change in data trend begins around $R e_{c}=110 \times 10^{3}, R e_{c}=125 \times 10^{3}$ and $R e_{c}=135 \times 10^{3}$ at $\alpha=0^{\circ}, 5^{\circ}$ and $10^{\circ}$, respectively, and takes place over a short range of Reynolds numbers until the separation bubble is formed (cf. figure 19 and table 1). The analysis of the results shows that, at a given angle of attack, the vortex shedding frequency increases linearly with the Reynolds number within each of the two data sets (figure 19a). In terms of the dimensionless shedding frequency, $F_{s}=f_{s} d^{2} / \nu$, introduced by Roshko (1954a), it can be shown that the dependency is of the form $F_{s} \sim R e_{c}$. Despite a significant difference in experimental parameters and flow geometry, a similar dependency was reported by Roshko (1954a) for the wake of a circular cylinder and by Huang et al. (2001) for laminar vortex shedding from a NACA 0012 airfoil at post-stall angles of attack (i.e. $\alpha \geqslant 12^{\circ}$ ). It can be seen in figure 19(a) that, for the lower Reynolds numbers corresponding to boundary layer separation without reattachment, the rate of increase of $f_{s}$ with $R e_{c}$ decreases with an increase of $\alpha$. This angle of attack effect diminishes significantly at higher Reynolds numbers, when the separation bubble is formed, but the rate of increase of $f_{s}$ with $R e_{c}$ is substantially higher for this flow regime. 


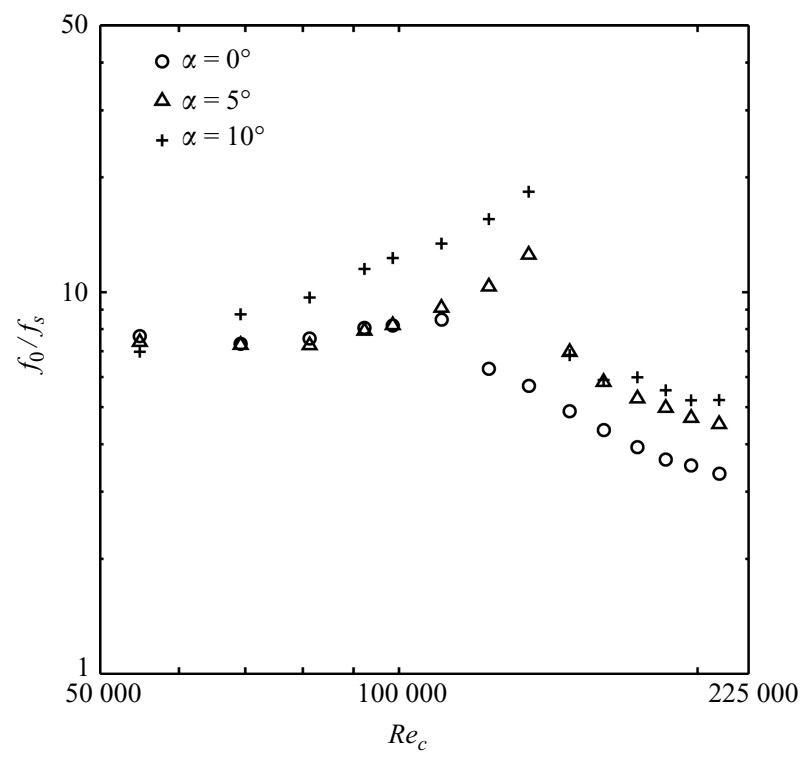

FigURE 20. Variation of the fundamental frequency normalized by the vortex shedding frequency $\left(f_{0} / f_{s}\right)$ with Reynolds number.

On the basis of the results in figure 19(b) or the established dependency between $F_{s}$ and $R e_{c}$, the Strouhal number varies with the Reynolds number as $S t_{s} \sim 1 / R e_{c}$. For the lower Reynolds numbers, at $\alpha=0^{\circ}, S t_{s}$ increases monotonically with $R e_{c}$ (figure 19b). At $\alpha=5^{\circ}$, an increase of $S t_{s}$ with $R e_{c}$ is checked, with $S t_{s}$ remaining approximately constant at 0.28 for $R e_{c}>80 \times 10^{3}$ until the boundary layer flow regime changes. In contrast to the two lower angles of attack, at $\alpha=10^{\circ}$, the Strouhal number is almost constant, with $S t_{s} \approx 0.2$. This suggests that the airfoil at this angle of attack behaves similar to a bluff body. The observed trends at lower Reynolds numbers are in agreement with those reported by Huang \& Lin (1995) for a NACA 0012 airfoil; however, as was discussed earlier, no evidence of vortex shedding at higher Reynolds numbers was revealed in their study. The present results show that, when the separation bubble is formed, the Strouhal number increases steadily with the Reynolds number at all three angles of attack, with lower angles of attack producing higher Strouhal numbers for a given $R e_{c}$. This flow regime is associated with much higher wake Strouhal numbers, so that the formation of the separation bubble has an effect similar to that of geometry modification to a more streamlined body, which is associated with an increase of $S t_{s}$ (e.g. Roshko 1954b).

For a circular cylinder, Bloor (1964) showed that a separated shear layer instability frequency normalized by a vortex shedding frequency varies as $f_{0} / f_{s} \sim\left(R e_{d}\right)^{m}$, where $R e$ is based on the cylinder diameter. This power-law relationship has been supported by the results of others (e.g. Prasad \& Williamson 1997; Thompson \& Hourigan 2005) with the value of the exponent $m$ varying; for example, $m=0.5$ (Bloor 1964) and $m=0.67$ (Prasad \& Williamson 1997). To investigate the possible existence of such a correlation for the case of an airfoil, the variation of $f_{0} / f_{s}$ with the Reynolds number is plotted on a logarithmic scale in figure 20 . For the lower Reynolds numbers, $f_{0} / f_{s}$ increases with an increase of Reynolds number. However, once the separation bubble forms on the airfoil surface, the normalized fundamental frequency decreases sharply. It then continues to decrease more gradually with an increase of Reynolds number. 


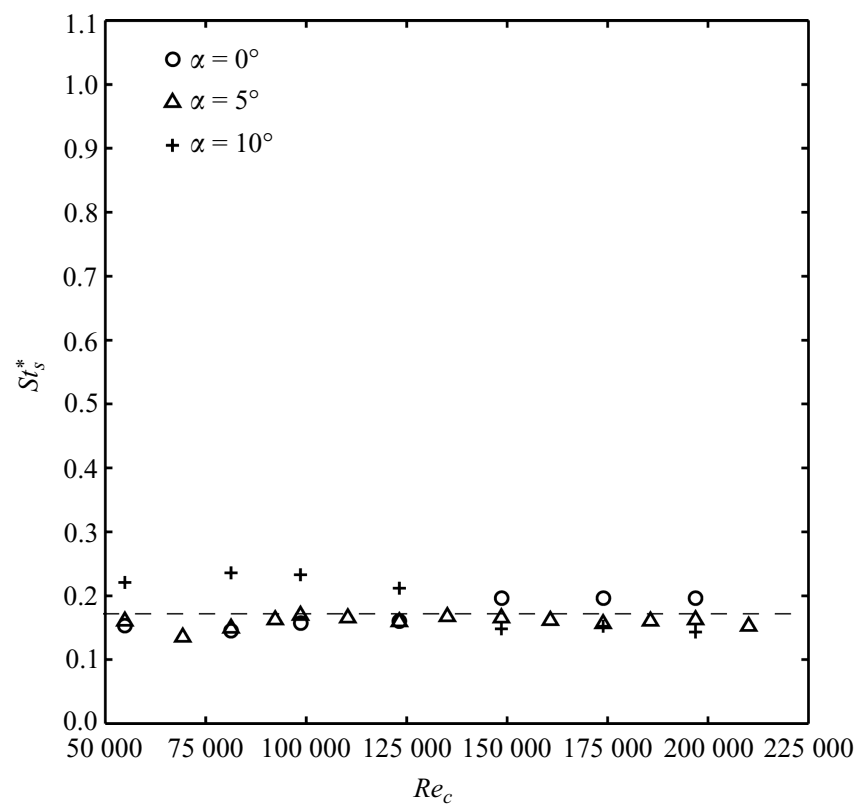

FIGURE 21. Universal vortex shedding frequency scaling based on wake flow geometry.

The data presented in figure 20 do not, in general, conform to a power-law relation. A reasonable power-law fit to the data can be made only for the lower Reynolds numbers at $\alpha=10^{\circ}$. This gives the exponent $m=1.04$, which is noticeably higher than the values reported for a circular cylinder.

\subsection{Universal frequency scaling}

Considering the substantial variation of flow characteristics within the investigated range of Reynolds numbers, a length scale linked to the wake vortex shedding phenomenon, as opposed to a geometric model dimension, would be a more appropriate scaling parameter for the vortex shedding frequency. Roshko (1954b) proposed a universal scaling of the vortex shedding frequencies in the wakes of bluff bodies based on the wake width. Following this approach, Roshko (1954b) obtained the universal Strouhal number of about 0.16 and Simmons (1977) suggested the value of 0.163 . In the present investigation, the vertical distance between two vortices forming in the near-wake region, $d^{*}$, is proposed as an alternative length scale. It was estimated as the distance between the two local maxima in the upper and lower portions of the wake r.m.s velocity profiles obtained at $x / c=1.25$ (Yarusevych 2006). The proposed parameter is not only linked to the investigated flow phenomenon but can also be determined more precisely compared to the wake width. The variation of the resulting 'universal' Strouhal number, $S t_{s}^{*}=f_{s} d^{*} / U_{0}$, with the Reynolds number is shown in figure 21. A comparison of the results presented in figure 19(b) and those in figure 21 shows that the proposed scaling dramatically reduces the variation of the scaled vortex shedding frequency. In fact, despite a minor scatter, the data collapse onto a universal Strouhal number $S t_{s}^{*} \approx 0.17$, which is in good agreement with that reported by Roshko (1954b) and Simmons (1977).

Although the characteristics of wake coherent structures depend strongly on the separated shear layer development, the observed similarity in the formation and arrangement of these structures, as well as the existence of the universal scaling 
for their shedding frequency, suggests that the two flow regimes are accompanied by the same vortex shedding phenomenon. Based on the similarity of the observed vortex shedding process to that typical of bluff-body wakes, it can be speculated that vortex formation in the airfoil wake at low Reynolds numbers is caused by a global instability in the near-wake region. It should be emphasized that these results pertain to turbulent wake vortex shedding. For laminar wake vortex shedding from an airfoil, a different mechanism was reported by Huang et al. (2001). A NACA 0012 airfoil was investigated for $R e_{c}<3000$, and the results show that vortices start to develop in the upper-side separated shear layer and at the trailing edge. These vortices continue to grow until they shed alternately into the wake. In agreement with the present findings, the results reported by Huang et al. (2001) suggest that bluff-body vortex shedding becomes more dominant with an increase of Reynolds number.

\section{Concluding remarks}

Development of coherent structures in the separated shear layer and wake of a NACA 0025 airfoil at low Reynolds numbers has been studied experimentally for a range of Reynolds numbers, $55 \times 10^{3} \leqslant R e_{c} \leqslant 210 \times 10^{3}$, and three angles of attack, $\alpha=0^{\circ}, 5^{\circ}$ and $10^{\circ}$. For all the cases examined, laminar boundary layer separation occurs on the upper surface of the airfoil, and two flow regimes were investigated: (i) boundary layer separation without reattachment and (ii) separation bubble formation.

The results show that the amplification of flow disturbance in the separated shear layer eventually results in shear layer roll-up. The resulting roll-up vortices are then shed at the frequency of the most amplified disturbances, i.e. the fundamental frequency. Following the roll-up process, the final stage of transition is associated with the growth of a sub-harmonic component in the velocity spectrum. The analysis indicates that this is attributed to the merging of the roll-up vortices, with the merged vortices eventually breaking down to smaller scales during transition. The breakdown process appears to be similar to that observed by Burgmann et al. (2008). Thus, a possible interaction of the roll-up vortices and wake structures can take place only at relatively low Reynolds numbers when shear layer transition occurs in the near-wake region. Although the observed transition mechanism was similar for the two investigated flow regimes, the sub-harmonic growth of fundamental disturbances becomes less pronounced when the separation bubble forms on the airfoil surface, suggesting that the proximity of the wall influences the nonlinear stage of the transition process in the separated shear layer.

Evidence of turbulent wake vortex shedding has been obtained for all cases examined, amending the results reported by Huang \& Lin (1995) and Huang \& Lee (2000). Wake vortices form in the near-wake region and are shed alternately on the upper and lower sides of the turbulent wake. The coherence and length scale of the wake vortices decrease significantly when the separation bubble forms on the upper surface. However, the observed similarities in wake development and the existence of a universal scaling for the vortex shedding frequency suggests that, for both flow regimes, the wake vortex shedding is attributed to the same phenomenon, viz., near-wake instability.

A detailed investigation of the identified coherent structures in the separated shear layer and airfoil wake suggests that their behaviour and characteristics depend strongly on the Reynolds number and the flow regime. In particular, the fundamental frequency of the roll-up vortices developing in the separated shear layer scales with the 
Reynolds number according to $f_{0} \sim\left(R e_{c}\right)^{n}$, and the wake vortex shedding frequency shows a linear dependency on the Reynolds number. The precise correlations depend on the angle of attack and differ for the cases of boundary layer separation without reattachment and separation bubble formation.

An alternative scaling for both the fundamental frequency and the wake vortex shedding frequency has been proposed. Scaling the fundamental frequency with the wavelength of the fundamental disturbances in the separated shear layer results in Strouhal numbers falling within $0.45 \leqslant S t_{0}^{*} \leqslant 0.5$. On the other hand, employing the vertical distance between two vortices forming in the near-wake region as the length scale leads to a universal scaling for the vortex shedding frequency. The resulting Strouhal number $S t_{s}^{*} \approx 0.17$ is in good agreement with that obtained for various bluff bodies by Roshko (1954b) and Simmons (1977).

\section{REFERENCES}

AbDalla, I. E. \& YANG, Z. 2004 Numerical study of the instability mechanism in transitional separating-reattaching flow. Intl J. Heat Fluid Flow 25, 593-605.

BLoor, M. S. 1964 The transition to turbulence in the wake of a circular cylinder. J. Fluid Mech. 19, 290-303.

Boiko, A. V., Grek, G. R., Dovgal, A. V. \& Kozlov V. V. 2002 The Origin of Turbulence in Near Wall Flows. Springer-Verlag.

Brendel, M. \& Mueller, T. J. 1988 Boundary-layer measurements on an airfoil at low Reynolds numbers. J. Aircr. 25, 612-617.

BRendel, M. \& Mueller, T. J. 1990 Transition phenomena on airfoils operating at low chord Reynolds numbers in steady and unsteady flow. In Numerical and Physical Aspects of Aerodynamic Flows IV (ed. T. Cebeci), pp. 333-344. Springer-Verlag.

Burgmann, S., Brucker, C. \& Schroder, W. 2006 Scanning PIV measurements of a laminar separation bubble. Exp. Fluids 41, 319-326.

Burgmann, S., Dannemann, J. \& Schroder, W. 2008 Time-resolved and volumetric PIV measurements of a transitional separation bubble on an SD7003 airfoil. Exp. Fluids 44, 602-622.

Burgmann, S. \& SChroder, W. 2008 Investigation of the vortex induced unsteadiness of a separation bubble via time-resolved and scanning PIV measurements. Exp. Fluids 45, 675-691.

Carmichael, B. H. 1981 Low Reynolds number airfoil survey. NASA CR 165803, Vol. I.

Dovgal, A. V., Kozlov, V. V. \& Michalke, A. 1994 Laminar boundary layer separation: instability and associated phenomena. Prog. Aerosp. Sci. 30 61-94.

GASTER, M. 1967 The structure and behaviour of separation bubbles. Tech. Rep. Reports and Memoranda No. 3595. Aeronautical Research Council, London.

Gerontakos, P. \& LeE, T. 2005 Near wake behind an airfoil with leading-edge flow control. J. Aircr. 42, 561-567.

GerRard, J. H. 1966 The mechanics of the formation region of vortices behind bluff bodies. J. Fluid Mech. 25, 401-413.

Haggmark, C. P., Bakchinov, A. A. \& Alfredsson, P. H. 2000 Experiments on a two-dimensional laminar separation bubble. Phil. Trans. R. Soc. Lond. A 358, 3193-3205.

Ho, C. \& Huerre, P. 1984 Perturbed free shear layers. Annu. Rev. Fluid Mech. 16, 365-424.

HsiaO, F. B., LiU, C. F. \& TANG, Z. 1989 Aerodynamic performance and flow structure studies of a low Reynolds number airfoil. AIAA J. 27, 129-137.

Huang, L. \& Ho, C. 1990 Small-scale transition in a plane mixing layer. J. Fluid Mech. 210, 475-500.

Huang, R. F. \& LEe, H. W. 2000 Turbulence effect on frequency characteristics of unsteady motions in wake of wing. AIAA J. 38, 87-94. 
HuAnG, R. F. \& Lin, C. L. 1995 Vortex shedding and shear-layer instability of wing at low-Reynolds numbers. AIAA J. 33, 1398-1403.

Huang, R. F., Wu, J. Y., Jeng, J. H. \& Chen, R. C. 2001 Surface flow and vortex shedding of an impulsively started wing. J. Fluid Mech. 441, 265-292.

Kawall, J. G., Shokr, M. \& Keffer, J. F. 1983 A digital technique for the simultaneous measurements of streamwise and lateral velocities in turbulent flows. J. Fluid Mech. 133, $83-112$.

LANG, M., Rist, U. \& WAGner, S. 2004 Investigations on controlled transition development in laminar separation bubble by means of LDA and PIV. Exp. Fluids 36, 43-52.

LeBlanc, P., Blackwelder, R. \& Liebeck R. 1989 A comparison between boundary layer measurements in a laminar separation bubble flow and linear stability theory calculations. In Proceedings of Low Reynolds Number Aerodynamics Conference, Notre Dame, IN (ed. T. J. Mueller), 189-205, Springer-Verlag.

Lin, J. C. M. \& Pauley, L. L. 1996 Low-Reynolds-number separation on an airfoil. AIAA J. 34, $1570-1577$.

Malkiel, E. \& MaYle, R. E. 1996 Transition in a separation bubble. ASME J. Turbomach. 118, $752-759$.

MarXen, O. \& Rist, U. 2005 Direct numerical simulation of nonlinear transitional stages in an experimentally investigated laminar separation bubble. In High Performance Computing in Science and Engineering '05 (ed. W. E Nagel, W. Jager \& M. Resch) pp. 103-117. SpringerVerlag.

Marxen, O., Rist, U. \& Wagner, S. 2004 Effect of spanwise-modulated disturbances on transition in a separated boundary layer. AIAA J. 42, 937-944.

McAuliffe, B. R. \& YARAS, M. I. 2005 Separation-bubble-transition measurements on a low-Re airfoil using particle image velocimetry.In Proceedingsof the ASME Turbo Expo 2005, Reno, NV, GT2005-68663.

MCAulifFe, B. R. \& YARAS, M. I. 2007 Transition mechanisms in separation bubbles under low and elevated free stream turbulence. In Proceedings of the ASME Turbo Expo 2007, Montreal, Canada, GT2007-27605.

Miksad, R. W. 1972 Experiments on the nonlinear stages of free shear layer transition. J. Fluid. Mech. 56, 695-719.

Mueller, T. J \& Delaurier, J. D. 2003 Aerodynamics of small vehicles. Annu. Rev. Fluid Mech. 35, 89-111.

Prasad, A. \& Williamson, C. H. K. 1997 The instability of the shear layer separating from a bluff body. J. Fluid Mech. 333, 375-402.

Roshкo, A. $1954 a$ On the development of turbulent wakes from vortex streets. NACA TR 1191.

Roshko, A. $1954 b$ On the drag and shedding frequency of two-dimensional bluff bodies. NACA TN 3169.

Roshko, A. 1993 Perspectives on bluff body aerodynamics. J. Wind Engng Ind. Aerodyn. 49, 79100.

Simmons, J. E. L. 1977 Similarities between two-dimensional and axisymmetric vortex wakes. Aeronaut. Q. 28, 15-20.

TANI, I. 1964 Low speed flows involving bubble separations. Prog. Aerosp. Sci. 5, 70-103.

Thompson, M. C. \& Hourigan, K. 2005 The shear-layer instability of a circular cylinder wake. Phys. Fluids 17 (021702), 1-4.

Unal, M. F. \& Rockwell, D. 1988 On vortex shedding from a cylinder. Part 1. The initial instability. J. Fluid Mech. 190, 491-512.

WATMUFF, J. H. 1999 Evolution of a wave packet into vortex loops in a laminar separation bubble. J. Fluid Mech. 397, 119-169.

Williams-Stuber, K. \& GHARIB, M. 1990 Transition from order to chaos in the wake of an airfoil. J. Fluid Mech. 213, 29-57.

Williamson, C. H. K. 1996 Vortex dynamics in the cylinder wake. Annu. Rev. Fluid Mech. 28, 477-539.

Wilson, P. G. \& Pauley L. L. 1998 Two- and three-dimensional large-eddy simulations of a transitional separation bubble. Phys. Fluids 10, 2932-2940. 
YANG, Z. \& VOKE, P. R. 2001 Large-eddy simulation of boundary-layer separation and transition at a change of surface curvature. J. Fluid. Mech. 439, 305-333.

YARUSEVYCH, S. 2006 Investigation of airfoil boundary layer and turbulent wake development at low Reynolds numbers. PhD thesis, University of Toronto, Canada.

Yarusevych, S., Sullivan, P. E. \& Kawall, J. G. 2006 Coherent structures in airfoil boundary layer and wake at low Reynolds numbers. Phys. Fluids 18 (044101), 1-11.

Zhang, W., HaIn, R. \& Kahler, J. 2008 Scanning PIV investigation of the laminar separation bubble on a SD7003 airfoil. Exp. Fluids 45, 725-743. 